\title{
Phase Precession Through Synaptic Facilitation
}

\author{
Kay Thurley* \\ thurley@pyl.unibe.ch \\ Institute for Theoretical Biology, Department of Biology, Humboldt-Universität \\ zu Berlin, 10115 Berlin, Germany
}

\section{Christian Leibold*}

leibold@bio.lmu.de

Institute for Theoretical Biology, Department of Biology, Humboldt-Universität zu Berlin, 10115 Berlin, Germany; Neuroscience Research Center, Charité, Universitätsmedizin Berlin, 10117 Berlin, Germany; and Bernstein Center for Computational Neuroscience, 10115 Berlin, Germany

\section{Anja Gundlfinger}

anja.gundlfinger@charite.de

Dietmar Schmitz

dietmar.schmitz@charite.de

Neuroscience Research Center, Charité, Universitätsmedizin Berlin, 10117 Berlin, Germany; and Bernstein Center for Computational Neuroscience, 10115 Berlin, Germany

\section{Richard Kempter}

r.kempter@biologie.hu-berlin.de

Institute for Theoretical Biology, Department of Biology, Humboldt-Universität zu Berlin, 10115 Berlin, Germany; Neuroscience Research Center, Charité, Universitätsmedizin Berlin, 10117 Berlin, Germany; and Bernstein Center for Computational Neuroscience, 10115 Berlin, Germany

Phase precession is a relational code that is thought to be important for episodic-like memory, for instance, the learning of a sequence of places. In the hippocampus, places are encoded through bursting activity of socalled place cells. The spikes in such a burst exhibit a precession of their firing phases relative to field potential theta oscillations $(4-12 \mathrm{~Hz})$; the theta phase of action potentials in successive theta cycles progressively decreases toward earlier phases. The mechanisms underlying the generation of phase precession are, however, unknown. In this letter, we show

*Kay Thurley is now at the Institute of Physiology, University of Bern, 3012 Bern, Switzerland. Christian Leibold is now at the Department of Biology II, LudwigMaximilians-Universität München, 82152 Planegg-Martinsried, Germany. 
through mathematical analysis and numerical simulations that synaptic facilitation in combination with membrane potential oscillations of a neuron gives rise to phase precession. This biologically plausible model reproduces experimentally observed features of phase precession, such as (1) the progressive decrease of spike phases, (2) the nonlinear and often also bimodal relation between spike phases and the animal's place, (3) the range of phase precession being smaller than one theta cycle, and (4) the dependence of phase jitter on the animal's location within the place field. The model suggests that the peculiar features of the hippocampal mossy fiber synapse, such as its large efficacy, long-lasting and strong facilitation, and its phase-locked activation, are essential for phase precession in the CA3 region of the hippocampus.

\section{Introduction}

Synapses typically change as a result of prior activation. Fully reversible synaptic changes that decay within about a minute are called short-term plasticity. This type of plasticity is prevalent in a variety of regions of the central nervous system (Zucker \& Regehr, 2002). Two major forms of synaptic short-term plasticity have been described: short-term depression goes along with an attenuation of the efficacy of synaptic transmission following the arrival of a spike; short-term facilitation describes an increase of the efficacy. Without further presynaptic activation, the efficacy recovers within characteristically hundreds of milliseconds, but recovery time constants of tens of seconds have also been reported, for example, for the hippocampal mossy fiber synapse (Salin, Scanziani, Malenka, \& Nicoll, 1996; Gundlfinger et al., 2007).

Only a few hypotheses on the functional role of short-term plasticity are available, despite the existence of various types of dynamical synapses in the central nervous system. Short-term depression is proposed, for instance, as a mechanism for an input-specific gain control (Abbott, Varela, Sen, \& Nelson, 1997), the detection of input coherence (Tsodyks \& Markram, 1997; Senn, Segev, \& Tsodyks, 1998), and encoding of stimulus features in the visual cortex (Artun, Shouval, \& Cooper, 1998; Buchs \& Senn, 2002). Short-term facilitation is discussed, for example, as a mechanism for temporal integration of presynaptic input (Buonomano \& Merzenich, 1995; Maass, Natschläger, \& Markram, 2002; Abbott \& Regehr, 2004). Here we propose that synaptic facilitation, for example, at the hippocampal mossy fiber (mf) synapse, allows for generating a relational spike code. This code might be important for one-shot learning and episodic-like memory, that is, the association of events in a behavioral sequence that occur on a timescale of seconds (Skaggs, McNaughton, Wilson, \& Barnes, 1996; Silva et al., 1996; Brun et al., 2002; Fortin, Agster, \& Eichenbaum, 2002; Kesner, Gilbert, \& Barua, 2002; Mehta, Lee, \& Wilson, 2002; Sato 
\& Yamaguchi, 2003; Melamed, Gerstner, Maass, Tsodyks, \& Markram, 2004; Jensen \& Lisman, 2005; Lengyel, Huhn, \& Erdi, 2005; Dragoi \& Buzsáki, 2006).

The hippocampal mossy fiber synapse is an extraordinarily large synapse that connects granule cells of the dentate gyrus (DG) to pyramidal cells of the CA3 region of the hippocampus (Henze, Urban, \& Barrionuevo, 2000). This synapse therefore participates in the trisynaptic hippocampal loop from the entorhinal cortex to the dentate gyrus, CA3, and CA1 (see Figure 1A). One of the peculiar features of the $\mathrm{mf}$ synapse is its tremendous short-term facilitation (see, e.g., Nicoll \& Schmitz, 2005, for a review). Following the arrival of a burst of spikes within a few seconds at the presynaptic terminal, the postsynaptic response amplitude can transiently increase severalfold (Salin et al. 1996; Toth, Suares, Lawrence, Philips-Tansey, \& McBain, 2000) such that a single presynaptic input spike can fire the pyramidal neuron in vitro (Jonas, Major, \& Sakmann, 1993) and in vivo (Henze, Wittner, \& Buzsáki, 2002). Furthermore, the facilitation decays with a time constant of about 10 seconds (Salin et al., 1996; Gundlfinger et al., 2007). Although the $\mathrm{mf}$ synapse has features that are, at least in its combination, unique in the central nervous system, its functional role in the hippocampal circuitry is still under debate. The classical point of view is that the mossy fiber synapse acts as a detonator that reliably transfers dentate activity into the cornu ammonis (McNaughton \& Morris, 1987; Urban, Henze, \& Barrionuevo, 2001). This detonator property has been exploited in models using the mossy fiber connections as teacher input (Treves \& Rolls, 1992) and to mediate context retrieval (Hasselmo \& Eichenbaum, 2005).

Hippocampal learning is linked to the so-called theta rhythm, which is a field potential oscillation in the frequency range of 4 to $12 \mathrm{~Hz}$ (see, e.g., Buzsáki, 2002, for a review). Theta oscillations are reliably observed when an animal explores its environment or during REM sleep. The activity of hippocampal neurons such as pyramidal cells of the Cornu ammonis (CA) and granule cells in the DG is phase-locked to theta oscillations (Skaggs et al., 1996). During the hippocampal theta state and when a rat is actively moving, CA pyramidal cells as well as DG granule cells show place-specific firing (O'Keefe \& Dostrovsky, 1971; Jung \& McNaughton, 1993; Skaggs et al., 1996). A receptive field of that kind is referred to as a place field. As a rat traverses a place field, the corresponding place cell emits a burst of spikes that typically lasts for a second, with maximum discharge rates of up to a few tens of spikes per second.

O'Keefe and Recce (1993) described a temporal fine structure in the spike bursts of hippocampal pyramidal cells during place field traversals. Determining the phases of the spikes in a burst with respect to theta oscillations of the field potential, the authors found that spikes in successive oscillation cycles occur at progressively earlier phases. They called this phenomenon hippocampal phase precession (see Figure 1B). A series of 
A

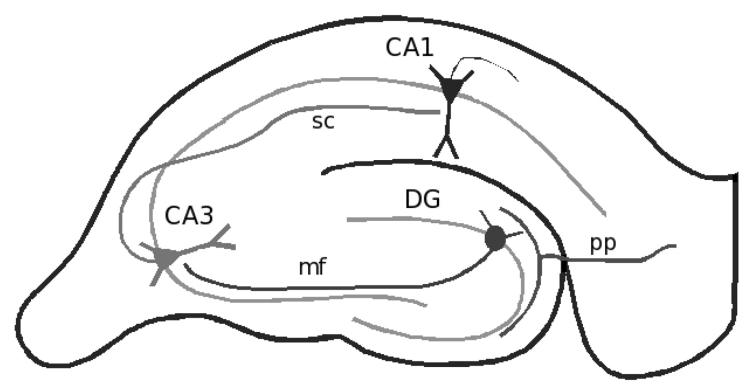

B
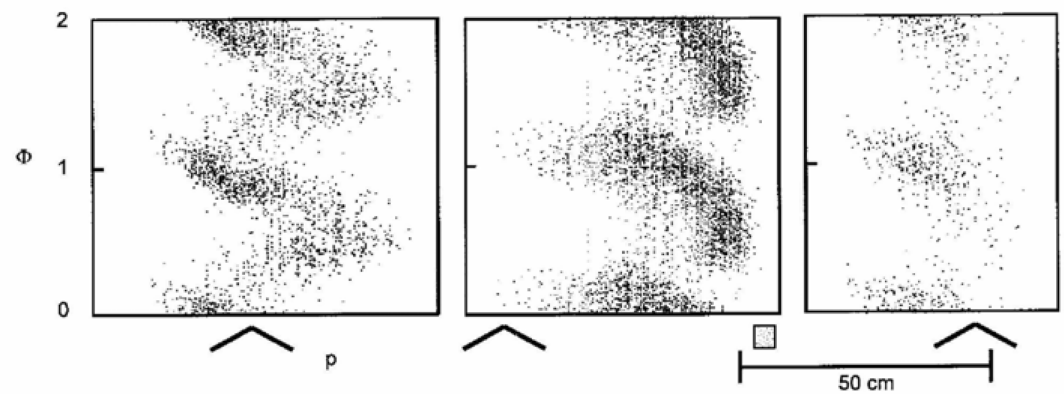

Figure 1: Hippocampal phase precession. (A) Trisynaptic hippocampal loop. Activity from superficial layers of the entorhinal cortex is conveyed to dentate gyrus (DG) granule cells via the perforant path (pp). Each granule cell contacts about 10 to 15 CA3 pyramidal cells by giant mossy fiber (mf) boutons sitting close to the soma. Axons of CA3 pyramidal cells give rise to recurrent connections within CA3, but also project via Schaffer collaterals (sc) to the pyramidal cells of area CA1. These subfields and pathways comprise the hippocampal trisynaptic loop (courtesy of Jörg Breustedt). (B) Phase precession in hippocampal place cells in vivo. The firing or output phase $\Phi$ of a pyramidal cell is plotted against the position $p$ of a rat freely moving from left to right. To account for the circular character of the firing phase $\Phi$, two cycles are shown. Dots represent single spikes. Boxes and arrows below the plots mark food locations and the corners of the triangular track used in the experiment, respectively. The horizontal extension of spikes represents the place field of the cell. The distribution of spike phases differs in the three cells. Phase precession can be bimodal (left panel) as well as curved (middle) or more linear (right). Adapted, with permission, from Skaggs et al. (1996).

subsequent papers has confirmed and refined this observation (e.g., Skaggs et al., 1996; Yamaguchi, Aota, McNaughton, \& Lipa, 2002; Harris et al., 2002; Mehta et al., 2002; Huxter, Burgess, \& O’Keefe, 2003; Dragoi \& Buzsáki, 2006; Maurer, Cowen, Burke, Barnes, \& McNaughton, 2006a; Chen \& Frank, 2007; 
see also the reviews by Maurer \& McNaughton, 2007, and Yamaguchi et al., 2007). Phase precession exists in both CA3 and CA1 pyramidal cells, but it is much less pronounced in CA1 interneurons (Maurer, Cowen, Burke, Barnes, \& McNaughton, 2006b; Ego-Stengel \& Wilson, 2007) and the dentate gyrus (Skaggs et al., 1996; Yamaguchi et al., 2002). Recently phase precession has been reported in layer II of the medial entorhinal cortex (Fyhn, Hafting, Moser, \& Moser, 2006), which provides input to the hippocampus. The origin of phase precession is still unknown, despite elegant experiments that aimed at unraveling underlying mechanisms (Ekstrom, Meltzer, McNaughton, \& Barnes, 2001; Huxter et al., 2003; Zugaro, Monconduit, \& Buzsáki, 2005), and despite reasonable effort to put forth mechanistic models (O'Keefe \& Recce, 1993; Tsodyks, Skaggs, Sejnowski, \& McNaughton, 1996; Jensen \& Lisman, 1996; Wallenstein \& Hasselmo, 1997; Bose \& Recce, 2001; Harris et al., 2002; Hasselmo, Canon, \& Koene, 2002; Mehta et al., 2002; Koene, Gorchetchnikov, Cannon, \& Hasslemo, 2003; Lengyel, Szatmary, \& Erdi, 2003; Yamaguchi, 2003; Huhn, Orban, Erdi, \& Lengyel, 2005; Scarpetta \& Marinaro, 2005; Lisman, Talamini, \& Raffone, 2005; Hasselmo \& Eichenbaum, 2005).

In this letter, we propose that synaptic facilitation in combination with oscillations of the membrane potential of the postsynaptic neuron accounts for phase precession. This novel and biologically plausible mechanism is in accordance with available experimental findings. In the next section, we illustrate the main idea through a minimal model. Section 3 formalizes this approach, provides some analytical results, and generalizes these results through numerical simulations. Section 4 explains the impact of various types of noise in our model of phase precession. Finally, in section 5, we simulate facilitating input impinging on conductance-based neurons during place field traversals.

\section{Precession of Firing Phases Through Facilitation of Synaptic Response Amplitudes}

The goal of this letter is to explain phase precession at the level of a single neuron at which facilitation of synaptic input and subthreshold oscillations of the membrane potential interact. In this section, we briefly outline the basic mechanism and the intrinsic features of the facilitation hypothesis of hippocampal phase precession.

2.1 Basic Mechanism. An important assumption of our model for phase precession is that neurons exhibit an oscillating excitability. An oscillating excitability is often taken into account through subthreshold oscillations of the membrane potential of a neuron at a constant firing threshold. Alternatively, one can assume a constant resting membrane potential and an oscillating firing threshold. In section 5, we show under which conditions both views are equivalent. For the time being and to explain the basic 

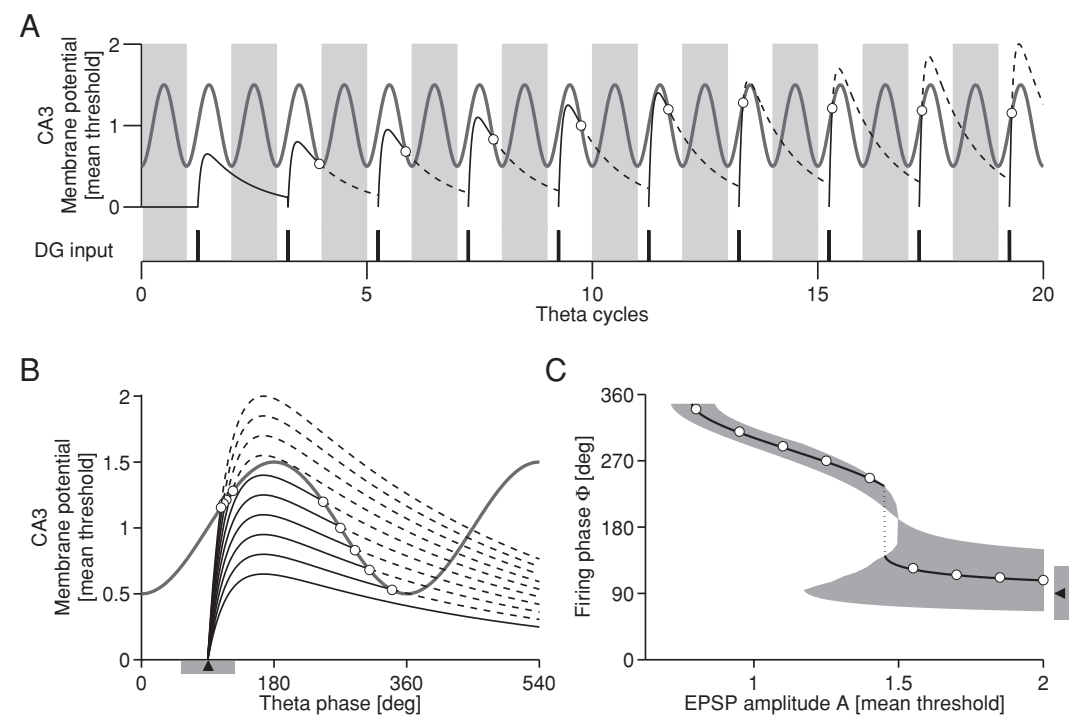

Figure 2: Phase precession evoked by synaptic facilitation. (A) We consider a CA3 pyramidal cell displaying a theta-modulated firing threshold (thick line; in units of the mean threshold). The cell receives DG input via a mossy fiber (mf). Every other theta cycle, we elicit one input spike at phase $\psi=90^{\circ}$. As the mf synapse facilitates, the EPSP amplitudes increase, and the phases of the evoked spikes (empty circles) decrease. Temporal summation of EPSPs has been neglected. (B) All theta cycles indicated in graph $A$ are mapped into one cycle. (C) The firing phase $\Phi$ of spikes in CA3 is a decreasing function of the EPSP amplitude $A$ (solid black line). Note the discontinuous phase change at about $A=1.4$. The empty circles correspond to the ones shown in graphs $A$ and $B$. The gray shaded area in $C$ marks the region of the $(A, \Phi)$ plane where we obtain spikes if the input phase $\psi$ is jittered over a range of $90 \pm 36^{\circ}$ (gray bars and arrowheads in $B$ and $C$ ).

mechanism of phase precession, we adopt the simplified view of an oscillating threshold. We define the minima of a sinusoidally oscillating firing threshold as 0 degree phase, which correspond to the maxima of a somatic membrane potential oscillation.

Further important assumptions of the model are facilitating synaptic input, the synaptic input being phase-locked to the oscillations, and synaptic input being strong enough to elicit an output spike in the postsynaptic neuron. Figure 2 sketches such a scenario for input that is phase-locked at 90 degrees. As a result, the output phase $\Phi$ at which an excitatory postsynaptic potential (EPSP) reaches the firing threshold strongly depends on the EPSP amplitude $A$. 
To describe the basic mechanism of our model of phase precession, we display EPSPs at various amplitudes in Figure 2B. The EPSP that is just sufficiently large to cross the oscillating threshold accounts for an output phase $\Phi$ close to, but below, the phase 360 degrees. A large EPSP, on the other hand, evokes an output spike almost instantaneously, with only little offset between the input phase $\psi=90^{\circ}$ and the output phase $\Phi$. While the EPSP amplitude $A$ increases, the firing phase $\Phi$ monotonically decreases (see Figure 2C). Note that in our example, with input phase $\psi=90^{\circ}$, the precession of $\Phi$ is discontinuous. How the choice of the input phase $\psi$ and all other model parameters affects phase precession is outlined below in detail.

\subsection{Hippocampal Phase Precession Through Mossy Fiber Facilitation.} We briefly review the biological feasibility of our main assumptions. Membrane potential oscillations, phase-locked synaptic input, and synaptic facilitation are the crucial components for the proposed mechanism of phase precession. These features have been described in detail in the CA3 region of the hippocampus. To be more specific, hippocampal pyramidal cells exhibit membrane potential oscillations in the theta range, which reflect the electroencephalogram (EEG) theta oscillations of the field potential. Intracellular theta oscillations have amplitudes of up to $10 \mathrm{mV}$ in anesthetized animals (Kamondi, Acsady, Wang, \& Buzsáki, 1998; Bland, Konopacki, \& Dyck, 2005), and were also observed in behaving animals (Lee, Manns, Sakmann, $\&$ Brecht, 2006). The maximum of the somatic membrane potential oscillation corresponds to the minimum of the EEG in the stratum pyramidale (Kamondi et al., 1998), which is defined as 0 degree in accordance with Csicsvari, Hirase, Czurkó, Mamiya, and Buzsáki (1999) and Buzsáki (2002).

Granule cells in the DG, which project to CA3 pyramidal cells, fire phaselocked to the theta rhythm (Ylinen et al., 1995; Skaggs et al., 1996). DG granule cells are also characterized through burstlike activity when the animal runs through their place fields (Jung \& McNaughton, 1993; Wiebe \& Stäubli, 1999; Skaggs et al., 1996).

CA3 pyramidal cells receive input from DG granule cells via hippocampal mf synapses, which show short-term facilitation that can increase EPSP amplitudes severalfold (Salin et al., 1996; Nicoll \& Schmitz, 2005), so that a single EPSP can become large enough to trigger an action potential (Jonas et al., 1993; Henze et al., 2002). Accordingly, the synapse has been referred to as a detonator (McNaughton \& Morris, 1987; Urban et al., 2001). Facilitation decays on a timescale of 10 seconds (Salin et al., 1996; Gundlfinger et al., 2007), which is longer than the time of about 1 second an animal takes to traverse a place field. Facilitation therefore progressively increases during typical place field traversals. Hence, we think that the basic assumptions of our model are reasonably well justified in the CA3 region of the hippocampus.

In other brain regions, for example, the entorhinal cortex (EC), phase precession might be generated in a similar way, but the specific features 
of phase precession depend on the local properties of synaptic facilitation and membrane potential oscillations. Although our model can be generalized to other brain regions, we primarily refer to the CA3 region of the hippocampus to illustrate phase precession.

In the following section, we define the above model for phase precession in mathematical terms, which allows an in-depth analysis of how model parameters affect its behavior, independent of any specific brain region.

\section{Generic Solutions to the Threshold Model}

In the previous section, we reported qualitative results obtained with a threshold model of a neuron that receives input from a facilitating synapse. To investigate in greater detail under which conditions this model can account for the phenomenon of phase precession, we now provide a more formal definition.

3.1 Definition of the Threshold Model. Oscillations of the resting membrane potential of a neuron are interpreted as a modulation of its firing threshold $\vartheta$ (see Figure 2). We write the threshold as

$$
\vartheta(\varphi):=\vartheta_{0}-\vartheta_{1} \cos \varphi
$$

for $\vartheta_{0}>\vartheta_{1}>0$ where $\vartheta_{0}$ is the mean threshold and $\vartheta_{1}$ is the amplitude of the threshold oscillation. The phase $\varphi=\omega t$ equals time $t$ multiplied by the circular frequency $\omega=2 \pi / T_{\theta}$ with the oscillation period $T_{\theta}$.

The simulated input from a synapse to the model neuron is assumed to evoke EPSPs that have a uniform shape but a variable amplitude $A$. In this section we assume that EPSPs always start from zero resting potential. This view is equivalent to considering the influence of a single EPSP only, that is, EPSPs do not sum up; serial correlations are discussed in section 5. Here, we define the EPSPs as

$$
\epsilon(\varphi):=A \mathcal{N}\left(\tau_{m}, \tau_{c}\right)\left[\exp \left(-\frac{\varphi}{\omega \tau_{m}}\right)-\exp \left(-\frac{\varphi}{\omega \tau_{c}}\right)\right] \Theta(\varphi)
$$

where the amplitude $A$ is to be changed through synaptic facilitation. The shape of $\epsilon$ is determined by the current time constant $\tau_{c}>0$ and the membrane time constant $\tau_{m}>\tau_{c}$, which are interpreted as time constants of rise and decay of the EPSP, respectively. The factor $\mathcal{N}\left(\tau_{m}, \tau_{c}\right)=$ $\left[\left(\tau_{c} / \tau_{m}\right)^{\tau_{c} /\left(\tau_{m}-\tau_{c}\right)}-\left(\tau_{c} / \tau_{m}\right)^{\tau_{m} /\left(\tau_{m}-\tau_{c}\right)}\right]^{-1}$ normalizes the EPSP such that its maximum value equals $A$. The function $\Theta(x)$ denotes the Heaviside step function, $\Theta(x)=1$ for $x \geq 0$, and $\Theta(x)=0$ otherwise. 
An EPSP $\epsilon$ that is elicited at some input phase $\psi$ evokes an output spike in the model neuron at the firing phase $\Phi$ at which the firing threshold $\vartheta>0$ is reached for the first time. In other words, the condition

$$
\vartheta(\Phi)=\epsilon(\Phi-\psi)
$$

must be satisfied. For generic choices of $\epsilon$, equation 3.3 is transcendental and thus can only be solved numerically. A current time constant $\tau_{c}=0$, however, permits analytical solutions to equation 3.3, which we provide in the next section. Numerical solutions for $\tau_{c}>0$ are outlined in section 3.3.

3.2 Solutions to the Threshold Model for $\tau_{c}=0$. To analytically solve phase precession within the framework of the threshold model in equation 3.3, we set the current time constant $\tau_{c}$ to zero. The EPSP in equation 3.2 then takes the shape of a decaying exponential $\epsilon(\varphi)=$ $A \exp \left[-\varphi /\left(\omega \tau_{m}\right)\right] \Theta(\varphi)$ with amplitude $A$. From Figure 2 we can guess that especially the input phase $\psi$ is a crucial parameter. In what follows, we specify three characteristic input phases $\psi^{\min }, \psi^{\mathrm{d}-\mathrm{c}}$, and $\psi^{\max }$ that separate three regions of qualitatively different behavior of phase precession (see Figure 3).

3.2.1 Minimum Input Phase $\psi^{\mathrm{min}}$. For input phases $\psi$ below some minimum input phase $\psi^{\mathrm{min}}$, phase precession cannot be observed. Let us therefore consider an EPSP that is elicited at the earliest possible input phase $\psi=0^{\circ}$, that is, at the minimum of the oscillating firing threshold. According to equation 3.3, EPSPs that are large enough to reach the threshold always do so at an output phase $\Phi=0^{\circ}$, whatever $A$. Figure 3A motivates the existence of some input interval $\left[0, \psi^{\mathrm{min}}\right]$, where phase precession is impossible because EPSPs decline too rapidly ever to reach the threshold in their decaying part. For $\psi=\psi^{\mathrm{min}}$, an EPSP that is just large enough to reach the threshold instantaneously at $\Phi=\psi$ also just reaches the threshold at a second phase $\Phi=\Phi^{\max }>\psi$. In this way, we define the maximum firing phase $\Phi^{\text {max }}$. The minimum phase $\psi^{\text {min }}$ cannot be given explicitly since equation 3.3 and $\epsilon\left(\Phi^{\max }\right)=A \exp \left[-\left(\Phi^{\max }-\psi^{\min }\right) /\left(\omega \tau_{m}\right)\right]$ only lead to the implicit expression

$$
\exp \left[\psi^{\min } /\left(\omega \tau_{m}\right)\right] \vartheta\left(\psi^{\min }\right)=\exp \left[\Phi^{\max } /\left(\omega \tau_{m}\right)\right] \vartheta\left(\Phi^{\max }\right),
$$

which allows numerically deriving the minimum phase $\psi^{\text {min }}$, given a fixed value of $\Phi^{\text {max }}$.

3.2.2 Maximum Input Phase $\psi^{\max }$. Phase precession is impossible for $\psi$ above $\psi^{\max }$. The phase $\psi^{\max }$ is identical to the maximum output phase $\Phi^{\max }$ that we just have defined as the phase where an exponentially decaying EPSP $\epsilon$ reaches, but does not cross, the threshold $\vartheta$ (see Figure $3 \mathrm{~A}$ ). Formally, 


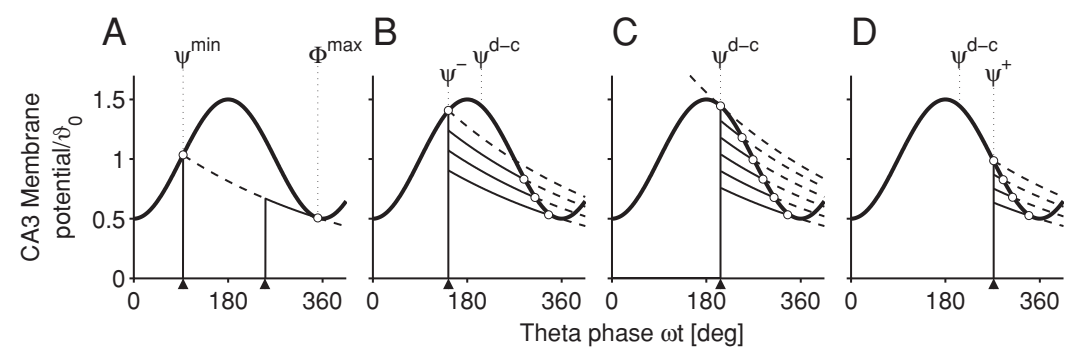

Figure 3: Phase precession for a current time constant $\tau_{c}=0$. Each graph shows an oscillating firing threshold (thick solid lines), exponentially decaying EPSPs (thin solid lines), phases of synaptic input (arrowheads), and the phases at which the EPSPs reach the threshold (empty circles). Dashed lines indicate the course of EPSPs beyond the point at which they have reached the threshold. (A) An EPSP that is just sufficiently large to reach the threshold in its decaying part evokes a spike at the maximum firing phase $\Phi^{\max }$. All other EPSPs that at least partly share this exponential function (dashed line) also reach the threshold at $\Phi^{\max }$. The earliest intersection between the exponential and the threshold (left-most empty circle) defines the minimum input phase $\psi^{\mathrm{min}}$. (B) For input phases $\psi$ with $\psi^{\mathrm{min}}<\psi<\psi^{\mathrm{d}-\mathrm{c}}$, for example, $\psi=\psi^{-}$, the output firing phase $\Phi$ is a discontinuous function of the EPSP amplitude; see also Figure 2C for finite $\tau_{c}$. (C) For an input phase $\psi=\psi^{\mathrm{d}-\mathrm{c}}$, we observe the largest range of continuous phase precession. (D) For input phases $\psi$ with $\psi^{\mathrm{d}-\mathrm{c}} \leq \psi<\psi^{\max }$, for instance, $\psi=\psi^{+}$, we have continuous phase precession. Further model parameters are $\tau_{m}=T_{\theta}$ and $\rho=\vartheta_{1} / \vartheta_{0}=0.5$.

$\Phi^{\max }$ is characterized by two conditions: the values and slopes of $\epsilon$ and $\vartheta$ are equal. From these conditions, the maximum firing phase is derived in appendix $\mathrm{A}$ as

$$
\Phi^{\max }=2 \pi-\arcsin \left\{\left[\rho \sqrt{1+\left(\omega \tau_{m}\right)^{2}}\right]^{-1}\right\}+\arctan \left[1 /\left(\omega \tau_{m}\right)\right],
$$

with $\rho:=\vartheta_{1} / \vartheta_{0}>1 / \sqrt{1+\left(\omega \tau_{m}\right)^{2}}$. We note that $\Phi^{\max }$ is independent of the input phase $\psi$ because EPSPs that just reach the threshold in their decaying part share the same exponential function, regardless of the input phase (see Figure 3A). Phase precession is therefore impossible for EPSPs initiated at phases $\psi \geq \Phi^{\max }$. Thus, $\Phi^{\max }$ is identical to the maximum input phase $\psi^{\max }$ that allows phase precession, that is, $\psi^{\max } \equiv \Phi^{\max }$.

3.2.3 Switch from Discontinuous to Continuous Phase Precession at Phase $\psi^{\mathrm{d}-\mathrm{c}}$. The interval ] $\psi^{\min }, \psi^{\max }$ [ of input phases $\psi$ that allow phase precession is divided into two intervals in which precession is either discontinuous or continuous. The two intervals are separated by the characteristic 


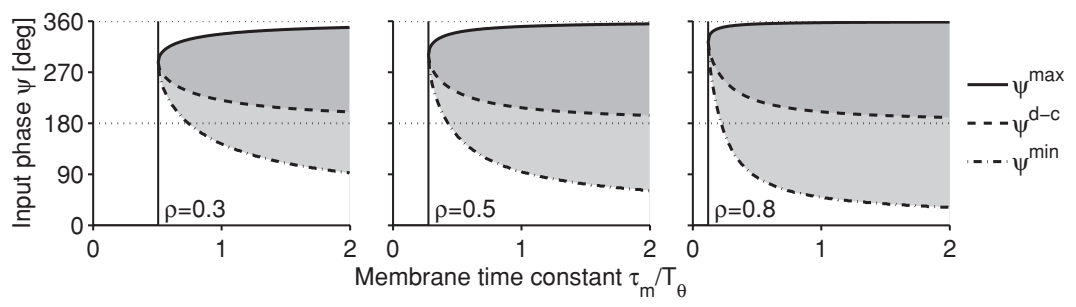

Figure 4: Continuous and discontinuous phase precession for three different modulation depths $\rho$ and for $\tau_{c}=0$. If the input phase $\psi$ is between $\psi^{\mathrm{d}-\mathrm{c}}$ (dashed line) and $\psi^{\max }$ (solid line), the output phase $\Phi$ is a continuous function of the EPSP amplitude $A$ (darker gray). Phase precession is discontinuous (lighter gray) for $\psi$ between $\psi^{\text {min }}$ (dot-dashed line) and $\psi^{\mathrm{d}-\mathrm{c}}$ (dashed line). The width of the two phase intervals increases with increasing both $\tau_{m} / T_{\theta}$ and $\rho:=\vartheta_{1} / \vartheta_{0}$. Outside the gray-shaded regions, phase precession is impossible. The minimum membrane time constant $\tau_{m}^{\min }$ from equation 3.7, below which the three characteristic phases do not exist, is marked by a solid vertical line. There we have $\psi^{\max }=\psi^{\mathrm{d}-\mathrm{c}}=\psi^{\min }=3 / 2 \pi+\arctan \left[1 /\left(\omega \tau_{m}^{\min }\right)\right]$.

phase $\psi^{\mathrm{d}-\mathrm{c}}$. Figure 3B indicates that for $\psi^{\mathrm{min}}<\psi<\psi^{\mathrm{d}-\mathrm{c}}$, the output phase $\Phi$ is a discontinuous function of the EPSP amplitude $A$. In contrast, for $\psi^{\mathrm{d}-\mathrm{c}} \leq \psi<\psi^{\max }$, the output phase $\Phi$ is a continuous function of $A$ (see Figures $3 C$ and $3 D$ ). The phase $\psi^{\mathrm{d}-\mathrm{c}}$ is determined similarly to $\psi^{\text {max }}$; we require that the values as well as the slopes of $\epsilon$ and $\vartheta$ be equal but that they are differently curved (see Figure 3C). In appendix A we show that these conditions lead to

$$
\psi^{\mathrm{d}-\mathrm{c}}=\pi+\arcsin \left\{\left[\rho \sqrt{1+\left(\omega \tau_{m}\right)^{2}}\right]^{-1}\right\}+\arctan \left[1 /\left(\omega \tau_{m}\right)\right]
$$

for $\rho \sqrt{1+\left(\omega \tau_{m}\right)^{2}} \geq 1$.

In summary, the case $\tau_{c}=0$ demonstrates that phase precession crucially depends on the input phase $\psi$. Phase precession is impossible for $0 \leq \psi \leq$ $\psi^{\mathrm{min}}$ and $\psi^{\max } \leq \psi<2 \pi$. Phase precession is discontinuous for $\psi^{\min }<\psi<$ $\psi^{\mathrm{d}-\mathrm{c}}$ and continuous for $\psi^{\mathrm{d}-\mathrm{c}} \leq \psi<\psi^{\max }$. In Figure 4 we have plotted $\psi^{\min }, \psi^{\max } \equiv \Phi^{\max }$, and $\psi^{\mathrm{d}-\mathrm{c}}$ as a function of $\tau_{m}$ for three values of the modulation depth $\rho$. The gray areas depict regions of the parameter space where phase precession can occur.

3.2.4 Minimum Values for $\tau_{m}$ and $\rho$ Are Required for Phase Precession. Let us assume, for the moment, that the modulation depth $\rho$ and the period $T_{\theta}$ are fixed. Figure 4 shows that the membrane time constant $\tau_{m}$ needs to exceed some minimal value $\tau_{m}^{\min }$ to allow phase precession. For $\tau_{m}>\tau_{m}^{\min }$, we obtain a nonvanishing interval $\left[\psi^{\min }, \psi^{\max }\right]$ of feasible input phases $\psi$. To 
calculate $\tau_{m}^{\mathrm{min}}$, we recall that phase precession needs dissimilar values for $\psi^{\mathrm{min}}, \psi^{\mathrm{d}-\mathrm{c}}$, and $\psi^{\mathrm{max}}$, which is guaranteed if the argument of the arcsin in equations 3.5 and 3.6 is within the open interval ]0, $1[$; that is, we require $\rho \sqrt{1+\left(\omega \tau_{m}\right)^{2}}>1$. From this condition, we find that $\tau_{m}$ needs to exceed

$$
\tau_{m}^{\min }:=\frac{\sqrt{1-\rho^{2}}}{\omega \rho} .
$$

In other words, every positive $\rho<1$ is associated with a lower bound $\tau_{m}^{\min }$ of the membrane time constant $\tau_{m}$; only for $\tau_{m}>\tau_{m}^{\min }$ is phase precession possible. As a corollary, we find that given $\tau_{m}$ and $\rho$, the period $T_{\theta}$ needs to be below $T_{\theta}^{\max }:=2 \pi \tau_{m} \rho / \sqrt{1-\rho^{2}}$. In a similar way we find that the modulation depth $\rho$ needs to exceed the value

$$
\rho^{\min }:=\frac{1}{\sqrt{1+\omega^{2} \tau_{m}^{2}}} .
$$

3.2.5 Iso- $\psi$ Lines and Phase Offset $\Phi-\psi$. Let us connect the analytical results obtained so far to Figure $2 \mathrm{C}$ where the firing phase $\Phi$ was plotted as a function of the EPSP amplitude $A$ for a constant input phase $\psi$. Such a graph is called an iso- $\psi$ line in the following.

Figure 5A shows numerically determined iso- $\psi$ lines for five different values of $\psi$, all for $\tau_{c}=0$. As expected, the three examples with input phases $\psi$ between $\psi^{\min }$ and $\psi^{\max }$ exhibit a maximum firing phase $\Phi^{\max }$ that is close to 360 degrees. The example with $\psi^{\min }<\psi<\psi^{\mathrm{d}-\mathrm{c}}$ indicates discontinuous phase precession, and the other two examples with $\psi^{\mathrm{d}-\mathrm{c}} \leq$ $\psi<\psi^{\max }$ demonstrate continuous phase precession. Figure 5A also verifies that the iso- $\psi$ lines are constant for large enough amplitudes $A$.

A remarkable property of iso- $\psi$ lines is that they can intersect. At the point of intersection of two iso- $\psi$ lines, the output phase $\Phi$ and the amplitude $A$ are identical, whereas the input phases $\psi$ are different. At slightly lower amplitudes, the EPSP that is elicited at the earlier input phase triggers a spike at a later firing phase; compare, for example, the iso- $\psi$ lines for $\psi=270^{\circ}$ and $207^{\circ}$ at amplitudes $A / \vartheta_{0} \lesssim 1$ in Figure 5A. Since for large enough amplitudes the firing phase $\Phi$ is always identical to the input phase $\psi$, iso- $\psi$ lines intersect at some intermediate amplitude.

To sum up the salient features of phase precession in one graph, we finally consider the phase offset $\Phi-\psi$, which is the difference between output and input phase. Figure 5B depicts $\Phi-\psi$ (in gray values) as a function of both the EPSP amplitude $A$ and the input phase $\psi$ for $\tau_{c}=0$. We find regions with no or only little phase precession, a transition from no phase precession to discontinuous phase precession at $\psi^{\mathrm{min}}$, and a transition from continuous to discontinuous phase precession at $\psi^{\mathrm{d}-\mathrm{c}}$. 


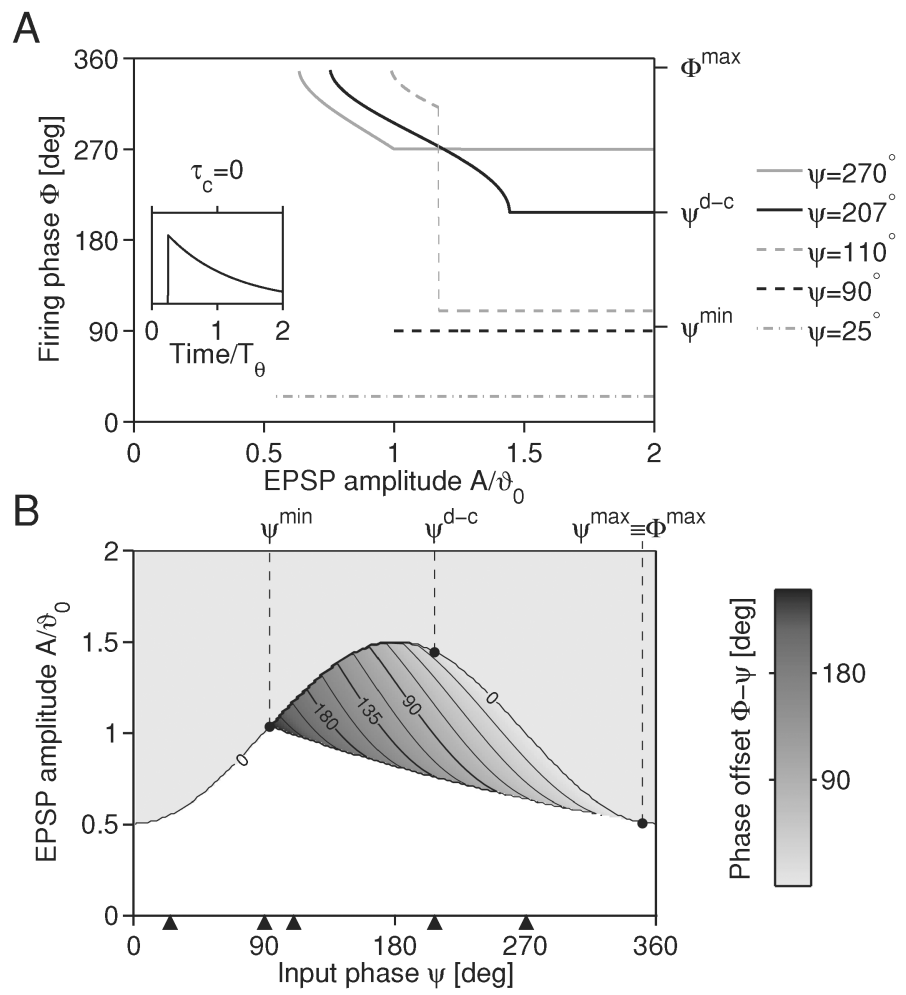

Figure 5: Iso- $\psi$ lines and phase offset $\Phi-\psi$ in the threshold model for $\tau_{c}=0$. (A) Iso- $\psi$ lines, that is, the firing phase $\Phi$ as a function of the EPSP amplitude $A$ (in units of the mean threshold $\vartheta_{0}$ ). The inset contains an example EPSP with instantaneous rise and exponential decay. We indicate the minimum input phase $\psi^{\text {min }}$ below which phase precession is impossible (see equation 3.4), the latest possible firing phase $\Phi^{\max }$ (see equation 3.5), and the minimum input phase for continuous phase precession $\psi^{\mathrm{d}-\mathrm{c}}$ (see equation 3.6). (B) Phase offset $\Phi-\psi$ (gray coded) as a function of the input phase $\psi$ and the EPSP amplitude $A$. For each input phase $\psi$, there exists a minimum amplitude below which the firing threshold $\vartheta$ is not reached (lower white region) and a maximum amplitude above which we always find $\Phi=\psi$ (upper lightest gray region); for intermediate amplitudes, gray values and contour lines every 22.5 degrees indicate the value of the phase offset $\Phi-\psi$. Phase precession occurs only for $\psi^{\min }<\psi<\psi^{\max }$. The phase offset $\Phi-\psi$ as a function of the amplitude $A$ has a discontinuity ("jump") for input phases $\psi^{\text {min }}<\psi<\psi^{\mathrm{d}-\mathrm{c}}$, whereas it is continuous for $\psi^{\mathrm{d}-\mathrm{c}} \leq \psi<\psi^{\max }$. The maximum phase offset amounts to about 251 degrees and is reached at $\psi=\psi^{\mathrm{min}}$. Arrowheads mark the input phases of the iso- $\psi$ lines in graph $A$. Other parameters are $\tau_{m}=T_{\theta}$ and $\vartheta_{1}=0.5 \vartheta_{0}$. 
To summarize, the special case $\tau_{c}=0$ of the oscillating-threshold model allowed us to derive conditions on the model parameters $\psi, \tau_{m}, \rho$, and $T_{\theta}$. These conditions considerably restrict the range of parameters at which we can observe phase precession through synaptic facilitation. Below we confirm that these restrictions can be transferred, at least qualitatively, to more complex models. As a next step, let us consider the case $\tau_{c}>0$.

3.3 Numerical Solutions to the Threshold Model for $\tau_{c}>0$. Important features of phase precession for a current time constant $\tau_{c}=0$ are preserved for $\tau_{c}>0$. To demonstrate the common features, we discuss numerical solutions of the threshold condition in equation 3.3 for $\tau_{c}>0$ and again leave membrane time constant $\tau_{m}=T_{\theta}$ and the threshold parameters $\vartheta_{0}=1$ and $\vartheta_{1}=0.5 \vartheta_{0}$ fixed.

For $\tau_{c}=0.075 T_{\theta}$, Figure $6 \mathrm{~A}$ contains iso- $\psi$ lines for the same five input phases $\psi$ that were used in Figure 5A for $\tau_{c}=0$. In both cases, iso- $\psi$ lines exhibit a variety of different shapes with a strong dependence on $\psi$; we show an example with little phase precession for early $\psi$ and examples with discontinuous as well as continuous phase precession. For the four largest values of $\psi$, the latest firing phase $\Phi^{\max }$ is close to 360 degrees, and the earliest firing phase is close to the input phase $\psi$. Finally, iso- $\psi$ lines can intersect.

The cases $\tau_{c}=0$ in Figure 5 and $\tau_{c}=0.075 T_{\theta}$ in Figure 6, however, also exhibit marked differences. For $\tau_{c}>0$, phase precession is always at least partly mediated by the rising part of the EPSP. This effect can be verified for large enough EPSP amplitudes $A$; with increasing $A$, the firing phase $\Phi$ slowly converges to the input phase $\psi$, which is reached only in the limit $A \rightarrow \infty$.

Increasing $\tau_{c}$ delays the peak of the EPSP, that is, shifts it to the right. This shift broadens the range of input phases $\psi$ that allow phase precession and also extends the maximum range of phase precession (given by the maximum phase offset). Moreover, increasing $\tau_{c}$ leads to a smoothing of the iso- $\psi$ lines, that is, an extension of the range of continuous phase precession.

The phase offset $\Phi-\psi$ in Figure 6B substantiates the existence of the characteristic phase $\psi^{\mathrm{d}-\mathrm{c}}$ for $\tau_{c}>0$, although its value can be determined only numerically. The minimum and maximum input phases $\psi^{\text {min }}$ and $\psi^{\max }$, however, do not exist for $\tau_{c}>0$. We can find an analog to the minimum input phase if we redefine $\psi^{\text {min }}$ to be the input phase where continuous phase precession switches to discontinuous phase precession (see appendix B). Then, for generic choices of $\epsilon$, the phase offset $\Phi-\psi$ for $0<\tau_{c} \ll T_{\theta}$ is homeomorphic to the case $\tau_{c}=0$. More precisely, there exists a continuous invertible mapping between the surfaces of phase offsets $\Phi(\psi, A)-\psi$ from Figures $5 \mathrm{~B}$ and 6B such that the "minimum" input phases $\psi^{\text {min }}$ and the input phases $\psi^{\mathrm{d}-\mathrm{c}}$ are mapped onto each other. The topologically invariant, and also most interesting, features of phase precession in our model can thus be understood in the case $\tau_{c}=0$. 


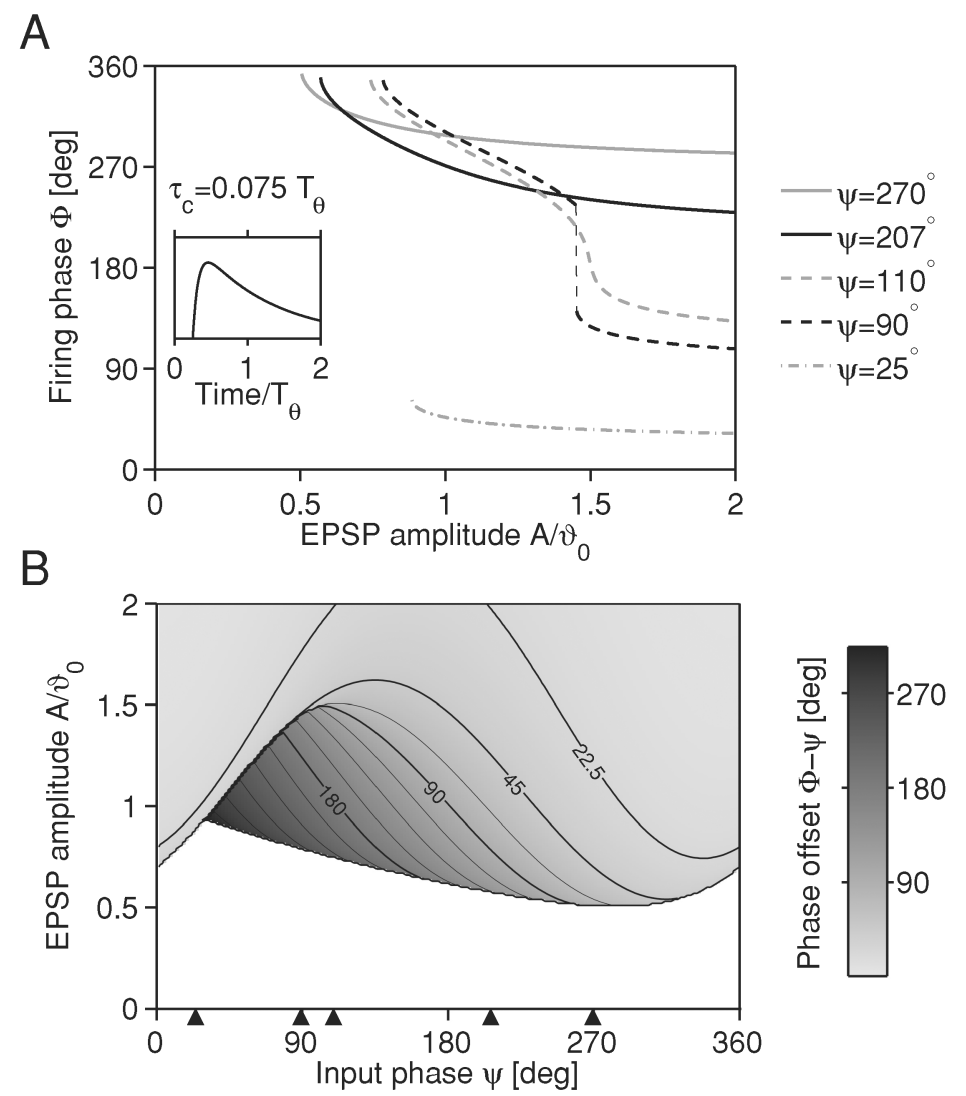

Figure 6: Iso- $\psi$ lines and phase offset $\Phi-\psi$ for $\tau_{c}=0.075 T_{\theta}$. (A) Iso- $\psi$ lines for the same input phases $\psi$ as in Figure 5. The inset depicts an example EPSP. For $\psi=25^{\circ}$, the small amount of phase precession between the latest firing phase of about 50 degrees and the earliest firing phase of about $\psi=25^{\circ}$ is entirely mediated by the rising part of the EPSP. The iso- $\psi$ line for $\psi=90^{\circ}$ shows discontinuous phase precession, whereas in Figure 5A for $\tau_{c}=0$, the same input phase led to only a constant firing phase $\Phi=\psi$. For $\psi=110^{\circ}$, we see continuous phase precession, whereas for the same $\psi$ but $\tau_{c}=0$, we had discontinuous phase precession. (B) Phase offset $\Phi-\psi$ (gray coded). Regions of continuous phase precession as well as discontinuities can be identified. The maximum phase offset of 317 degrees is reached at $\psi \approx 30^{\circ}$. Input phases $\psi$ that generate the iso- $\psi$ lines in graph $A$ are marked by arrowheads. Further parameters are $\tau_{m}=T_{\theta}$ and $\vartheta_{1}=0.5 \vartheta_{0}$. 
To conclude, we have outlined how the main features of phase precession depend on the parameters of a model in which progressively facilitating EPSPs intersect a sinusoidally oscillating firing threshold. Considering exponentially decaying EPSPs with a current time constant $\tau_{c}=0$ enabled a quantitative analysis. Results for $\tau_{c}=0$ also hold in the case of small $\tau_{c}$. For $\tau_{c}=0$, the output phase $\Phi$ is a decreasing function of the EPSP amplitude $A$ if the membrane time constant $\tau_{m}$ and the modulation depth $\rho$ of the firing threshold exceed some minimum values. We also saw that the output phase $\Phi$ is always between the input phase $\psi$ and some maximum firing phase $\Phi^{\max }$, which was typically close to 360 degrees. Phase precession thus depends critically on the input phase $\psi$. Varying $\psi$ revealed a transition from continuous to discontinuous phase precession at the minimal input phase $\psi^{\mathrm{min}}$ and a transition from discontinuous back to continuous phase precession at the phase $\psi^{\mathrm{d}-\mathrm{c}}$. To display further properties of phase precession, we now consider the influence of noise.

\section{Impact of Noise on Phase Precession}

To account for features of in vivo phase precession, we evaluate the influence of different sources of noise in the threshold model as defined in the previous section. In particular, we model noise in the input phase $\psi$, noise in the time course of the intracellular oscillation $\vartheta$, and noise in the mean firing threshold $\vartheta_{0}$.

4.1 Noise in the Input Phase $\psi$. Let us return to the specific example of phase precession in the CA3 region of the hippocampus. The activity of DG granule cells has been demonstrated to be theta-modulated, that is, spikes are phase-locked to the theta oscillations with some jitter (Ylinen et al., 1995; Skaggs et al., 1996). DG granule cells provide direct input to CA3 pyramidal cells via the $\mathrm{mf}$ synapses. Hence, the jitter of the firing phases of DG granule cells reflects the phase jitter of the input phase $\psi$ of mf EPSPs of our model.

Formally, we write the input phase jitter as $\psi \rightarrow \psi+\eta_{\psi}$, where $\eta_{\psi}$ is a zero mean random variable, for instance, gaussian or uniform, with standard deviation $\sigma_{\psi}$. The resulting distribution of output phases $\Phi$ of the model neuron can then be visualized through a combination of noisefree iso- $\psi$ lines. Figure 7A highlights the region of output phases that can be reached by input phases $\psi$ that vary between $90^{\circ}-36^{\circ}$ and $90^{\circ}+36^{\circ}$ (see also Figure 2C). Output spikes evoked by the lowest EPSP amplitudes $A$, for example $A / \vartheta_{0}=0.8$, occur close to 360 degrees with a relatively weak dependence on the input phase; the distribution of output phases $\Phi$ is narrower than the one of input phases $\psi$. For large EPSP amplitudes, for instance, $A>1.7 \vartheta_{0}$ (see Figure 7A), the output phase $\Phi$ converges to the input phase, and consequently, the jitter of the output approaches the jitter $\sigma_{\psi}$ of the input. For intermediate $A$ near the phase discontinuity at $A / \vartheta_{0}=1.5$, we observe a complex shape of the distribution of $\Phi$, which resembles 
A

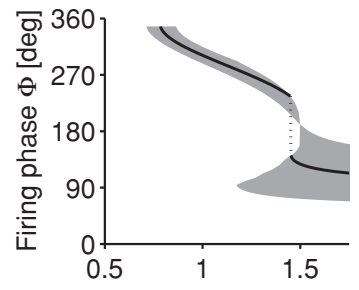

B

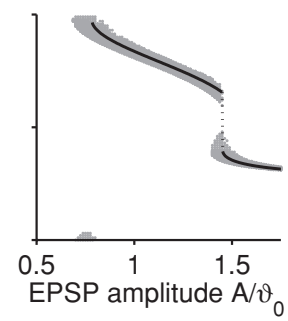

C

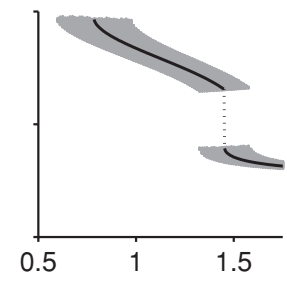

Figure 7: Variability of the firing phase for three different sources of noise. The three graphs show identical iso- $\psi$ lines for $\psi=90^{\circ}$ (solid lines), but different regions (gray-shaded) where output spikes are possible given a specific type of noise. (A) Noise in the input phase $\psi$ with jitter $\sigma_{\psi}=36^{\circ}$. The jitter in the output phase $\Phi$ is largest for large amplitudes $A$ (identical to Figure 2C). (B) Noise in the time course of the firing threshold $\vartheta$. The threshold jitter $\eta$ is uniformly distributed in the interval $\left[-0.05 \vartheta_{0}, 0.05 \vartheta_{0}\right]$ and updated at the sampling rate $100 / T_{\theta}$. This noise reduces the phase discontinuity, and output phases below 360 degrees become possible. The dependence of the output jitter on $A$ is weak. (C) Noise in the mean firing threshold $\vartheta_{0}$. The threshold jitter $\eta_{\vartheta}$ is uniformly distributed in the interval $\left[-0.125 \vartheta_{0}, 0.125 \vartheta_{0}\right]$ and updated once for an input spike. The distribution of output phases is expanded horizontally in the direction of the amplitude $A$. Other parameters in the three graphs are $\tau_{c}=0.075 T_{\theta}$, $\tau_{m}=T_{\theta}$, and $\vartheta_{1}=0.5 \vartheta_{0}$.

in vivo data (Skaggs et al., 1996; Yamaguchi et al., 2002; Mehta et al., 2002); see also Figure 1B.

4.2 Noise in the Time Course of the Firing Threshold $\vartheta$. Spiking activity of neurons is influenced by fluctuations in the intracellular oscillations. Within the framework of the threshold model, a noisy oscillation is realized through a noisy firing threshold,

$$
\vartheta(\varphi)=\vartheta_{0}+\vartheta_{1} \cos (\varphi)+\eta(\varphi)
$$

where $\eta$ is a random variable with zero mean and standard deviation $\sigma_{\eta}$. In Figure $7 \mathrm{~B}$, the variable $\eta$ is uniformly distributed in the interval $\left[-0.05 \vartheta_{0}, 0.05 \vartheta_{0}\right]$ and updated at the sampling rate of $100 / T_{\theta}$, which corresponds to $1 \mathrm{kHz}$ for $T_{\theta}=100 \mathrm{~ms}$. We chose these parameters to obtain a jitter in the output phase $\Phi$ that is similar to the one obtained in Figure 7A for small $A$. For large $A$, the situation is different; noisy oscillations then have relatively little impact on the output jitter.

4.3 Noise in the Mean Firing Threshold $\boldsymbol{\vartheta}_{0}$. A fluctuating mean threshold $\vartheta_{0}$ of firing is another possibility to introduce noise into the model. 

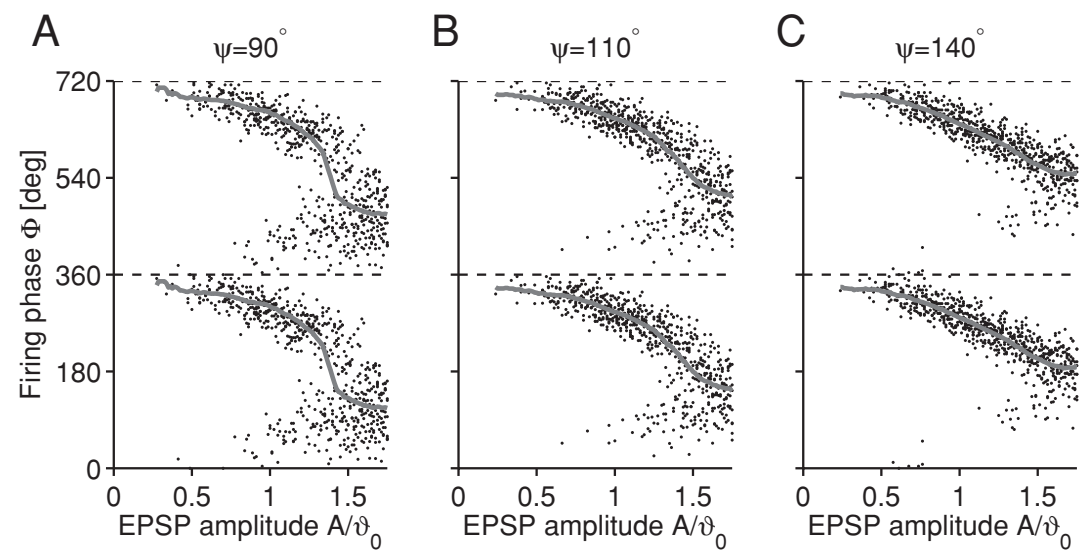

Figure 8: Combination of different sources of noise and the influence of the input phase on phase precession. Gaussian noise is concurrently added to the input phase $\left(\sigma_{\psi}=36^{\circ}\right)$, the theta oscillation $\left(\sigma_{\eta}=0.025 \vartheta_{0}\right.$, sampling rate $\left.100 / T_{\theta}\right)$, and the mean firing threshold $\left(\sigma_{\vartheta}=0.125 \vartheta_{0}\right)$ of the threshold model. Gray lines indicate the circular mean of output phase of spikes (dots). (A) Bimodal phase precession is observed for an input phase $\psi=90^{\circ}$. (B) Curved (nonlinear) phase precession occurs for $\psi=110^{\circ}$. (C) Linear phase precession is found for $\psi=140^{\circ}$. Further parameters of the threshold model are as in Figures 6 and 7: $\tau_{c}=0.075 T_{\theta}, \tau_{m}=T_{\theta}$, and $\vartheta_{1}=0.5 \vartheta_{0}$.

Formally, we add a zero mean random variable $\eta_{\vartheta}$ with standard deviation $\sigma_{\vartheta}$ to the threshold,

$$
\vartheta(\varphi)=\vartheta_{0}+\vartheta_{1} \cos (\varphi)+\eta_{\vartheta}
$$

For every evoked EPSP, the mean firing threshold assumes a different random value. In fact, equation 4.2 is a special case of equation 4.1 describing a slow variation of the mean firing threshold. In Figure 7C, $\eta_{\vartheta}$ is uniformly distributed in the interval $\left[-0.125 \vartheta_{0}, 0.125 \vartheta_{0}\right]$; such a noisy threshold introduces mainly a horizontal expansion of the phase distribution.

4.4 Influence of the Mean Input Phase on the Impact of Noise. So far we have considered effects of only a single source of noise at a time. Figure 8 shows spikes of the model neuron when the three different noise sources are combined. To demonstrate the influence of the mean input phase $\psi$ on phase precession in the presence of noise, we discuss the examples $\psi=90^{\circ}, \psi=110^{\circ}$, and $\psi=140^{\circ}$. For these three input phases, the firing phases $\Phi$ are distributed over almost the whole theta cycle, whereas the mean firing phase changes about 180 degrees. Note that the change in the 
mean firing phase is also described by the phase offset $\Phi-\psi$ in section 3; its maximum is the range of phase precession, and noise extends the range of spike phases. The amount of this extension depends on the amplitude $A$.

In Figure 8, the output phase distribution expands vertically with increasing amplitude $A$. Bimodality of the spike phase distribution, that is, two spike clusters, can be seen for $\psi=90^{\circ}$. A less bimodal spike distribution with a nonlinear, or curved, relation between phase and amplitude is obtained for $\psi=110^{\circ}$. Finally, for $\psi=140^{\circ}$, we find almost linear phase precession. Such variable shapes of phase-place distributions have also been observed in vivo; see, for example, Figure 1B and $\mathrm{O}^{\prime}$ Keefe and Recce (1993), Skaggs et al. (1996), Yamaguchi et al. (2002), Mehta et al. (2002), and Huxter et al. (2003).

In conclusion, our model indicates that the gross shape of phase distributions is sensitive to the preferred phase of the input. Furthermore, phase distributions are blurred by noise in a characteristic way. We note that a variable shape of the EPSP and other cellular heterogeneities as well as fluctuations in the oscillation frequency or amplitude can also contribute to the shape of phase-amplitude distributions. Systematically evaluating the latter dependencies is, however, beyond the scope of this letter. Instead, we are now continuing by testing the robustness of our model; therefore, we introduce a more involved description of a neuron, and we also connect the amplitude of EPSPs to the spatial position of a simulated animal.

\section{Conductance-Based Neurons and Place Fields}

The threshold model of phase precession, as discussed in the previous sections, can explain features of experimental data in the CA3 region of the hippocampus. The threshold model has, however, two restrictions. First, it cannot account for serial correlations between CA3 spikes. Serial correlations perturb the initial condition of postsynaptic integration, which might destroy or at least deteriorate precession of the firing phase $\Phi$. Second, the input of the model does not resemble in vivo-like activity patterns of dentate gyrus granule cells during the traversal of place fields. To verify that above restrictions are not crucial for our model of phase precession, we utilize a conductance-based leaky integrate-and-fire neuron in section 5.1, and introduce a Poisson model of place cell-like firing of DG granule cells in section 5.2.

5.1 Phase Precession Despite Serial Correlations. The subthreshold dynamics of the membrane voltage $V$ of a leaky integrator is given by the differential equation

$$
\frac{\mathrm{d} V}{\mathrm{~d} t}=-\frac{V}{\tau_{m}}+g_{\mathrm{mf}}(t)\left(E_{\mathrm{mf}}-V\right)+g_{\theta}(t)\left(E_{\theta}-V\right)+I_{\mathrm{DC}}
$$


which introduces the reversal potentials $E_{\mathrm{mf}}$ and $E_{\theta}$ as well as the synaptic conductances $g_{\mathrm{mf}}(t)$ and $g_{\theta}(t)$ of the excitatory $\mathrm{mf}$ input and inhibitory input, respectively (Troyer \& Miller, 1997). The constant current $I_{\mathrm{DC}}$ determines the resting potential. An output spike is emitted when the membrane potential $V$ reaches the firing threshold $\vartheta$. Immediately after a spike, the membrane

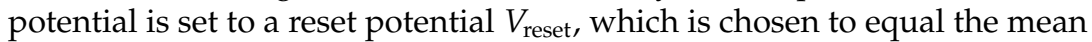
of the membrane potential oscillation.

The inhibitory conductance $g_{\theta}$ is assumed to be the origin of the theta oscillations of the membrane potential. This conductance is therefore modeled as

$$
g_{\theta}(t)=\gamma_{0}+\gamma_{1} \cos (\omega t)
$$

with parameters $\gamma_{0}$ and $\gamma_{1}$, which correspond to the parameters $\vartheta_{0}$ and $\vartheta_{1}$ of the threshold model in equation 3.1, respectively. Similarly, the conductance $g_{\mathrm{mf}}(t)$ replaces the $\mathrm{mf}$ EPSP $\epsilon(\varphi)$ in equation 3.2. Assuming $T_{\theta}$-periodic granule cell firing at an input phase $\psi$, we describe the time course of $g_{\mathrm{mf}}$ by the differential equation

$$
\frac{\mathrm{d} g_{\mathrm{mf}}}{\mathrm{d} t}=-\frac{g_{\mathrm{mf}}}{\tau_{c}}+\sum_{f=1}^{F} \gamma_{\mathrm{mf}}^{(f)} \delta\left(t-\psi / \omega-f T_{\theta}\right),
$$

where $\gamma_{\mathrm{mf}}^{(f)}$ is the amplitude of the $f$ th $\mathrm{mf}$ input and $F$ is the number of theta cycles within the place field. The symbol $\delta$ denotes the Dirac distribution. Hence, the excitatory input to the integrate-and-fire neuron consists of one spike per cycle. Furthermore, the conductance $g_{\mathrm{mf}}$ is set to zero immediately after an output spike of the neuron. We therefore avoid multiple spikes of the model neuron in response to a single input spike. This restriction allows a better comparison with the threshold model.

Facilitating $\mathrm{mf}$ input is modeled through a linear increase $\Delta$ of the amplitude for each input spike in addition to the baseline amplitude $\gamma$. The $f$ th amplitude then is $\gamma_{\mathrm{mf}}^{(f)}=\gamma+f \Delta$. Decay of the facilitation is neglected since $\mathrm{mf}$ facilitation characteristically lasts for several seconds, that is, $\mathrm{mf}$ facilitation decays on a timescale much longer than the traversal of a place field. Depending on the number $F$ of input spikes, $\gamma$ and $\Delta$ are to be chosen such that phase precession occurs over the whole place field.

Simulations of the conductance-based neuron substantiate that serial correlations between successive output spikes do not prevent phase precession. Figure 9 depicts a sample trace of a solution to equation 5.1 in the noise-free case. The parameters $\gamma_{0}$ and $\gamma_{1}$ are set such that the resulting subthreshold oscillations of the membrane potential resemble the situation in Figure 2A if we consider synaptic integration to start at the reset potential $V_{\text {reset }}=0.6 \vartheta$. The phase of granule cell input has been set to $\psi=110^{\circ}$. In accordance with the simple threshold model in section 3, action potentials elicited by EPSPs that are just sufficiently large to cross the threshold are 


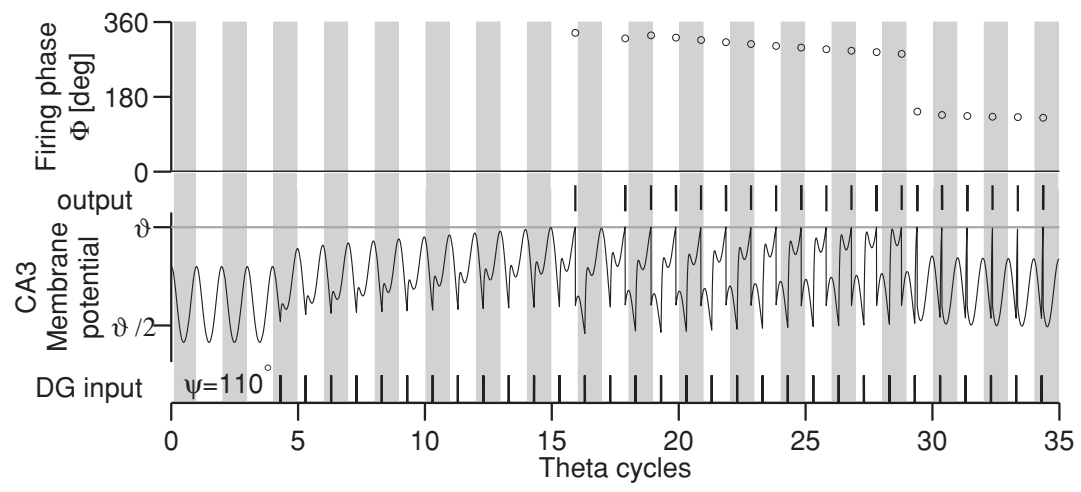

Figure 9: Phase precession in a conductance-based neuron. The model neuron receives one DG input spike per cycle at phase $\psi=110^{\circ}$ (bottom). The membrane potential of the simulated CA3 neuron and the evoked output spikes are depicted in the middle and top panels, respectively. The firing phase $\Phi$ precesses as the synaptic input facilitates. Here, phase 360 degrees corresponds to the peaks of the membrane potential oscillation without facilitating input in the first four theta cycles. Other parameters are chosen such that the scenario is similar to Figures 2 and 6. In detail, we have $\tau_{c}=0.075 T_{\theta}, \tau_{m}=T_{\theta}, E_{\mathrm{mf}}=2 \vartheta$, $\gamma=0.027, \Delta=0.003, \gamma_{0}=\gamma_{1}=0.014, E_{\theta}=-1 / 3 \vartheta, I_{\mathrm{DC}}=1.85 \vartheta / \tau_{m}$.

located at the peaks of the intracellular subthreshold oscillations. These peaks correspond to minimum inhibition and are defined as 360 degrees. For strongly facilitated mf EPSPs, firing occurs with little offset between input phase $\psi$ and firing phase $\Phi$. In the example with $\psi=110^{\circ}$, spikes are evoked in the troughs of the membrane potential oscillation.

Let us now investigate the influence of noise on phase precession in a conductance-based neuron. In Figure 10, we added three sources of noise, as described in section 4. Results resemble the ones shown in Figure 8: spikes are distributed over the whole theta cycle, and the mean firing phase decreases over more than 180 degrees. Again, depending on the input phase $\psi$, we find bimodal $\left(\psi=90^{\circ}\right)$, curved $\left(\psi=110^{\circ}\right)$, or more linear $\left(\psi=140^{\circ}\right)$ spike phase distributions. In single trials, however, phase precession might be hard to detect (see Figure 10A).

In this section, we established that phase precession is robust not only to temporal summation of inputs but also to serial correlations of spiking activity in the model neuron. Stimulating a conductance-based integrate-andfire neuron with periodic input spikes and introducing noise, we obtained results that are similar to the threshold model in the previous sections. The stimuli applied so far, however, do not resemble granule cell activity during the traverse of a place field. Furthermore, the relation between firing phase and the spatial location of a simulated rat remains unspecified. Both issues are addressed in the next section. 

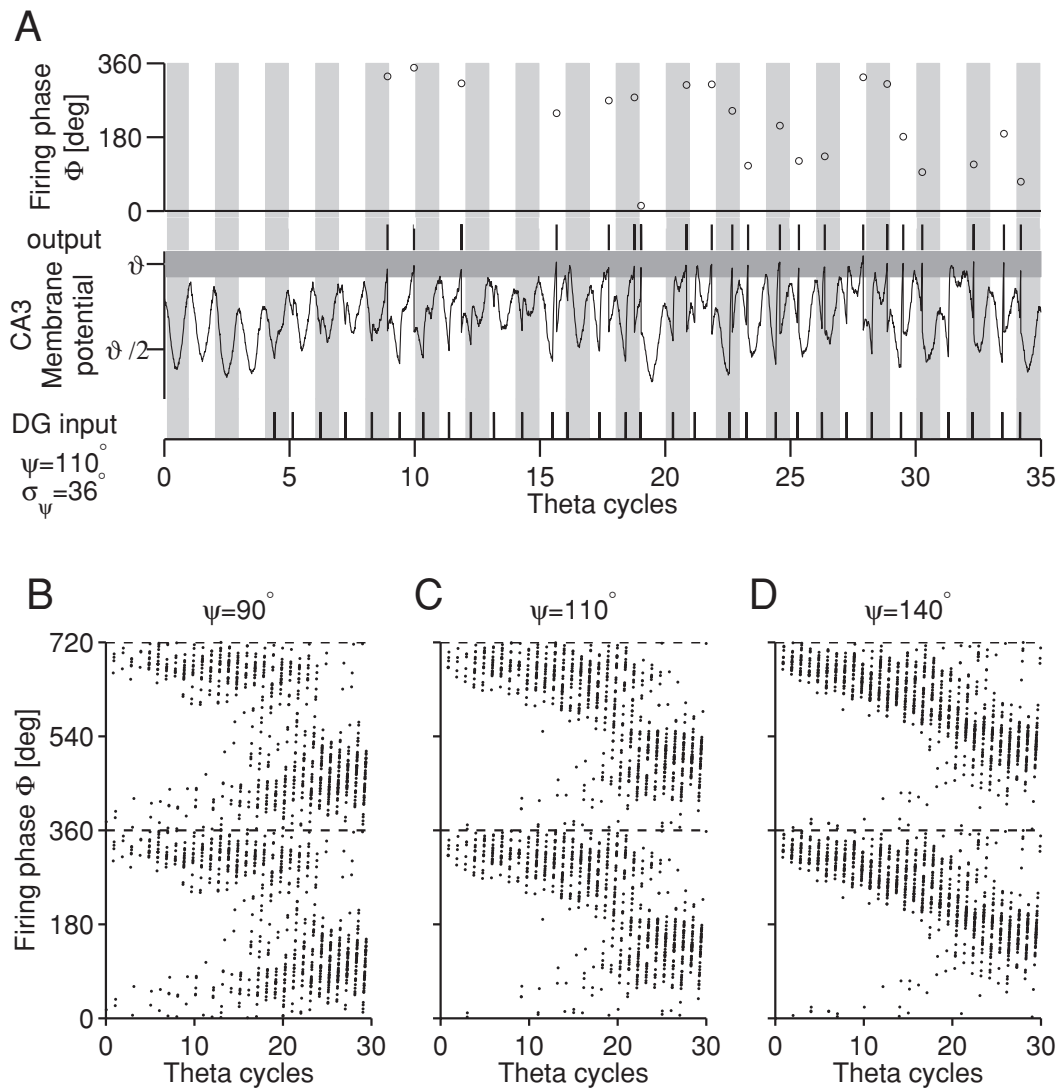

Figure 10: Phase precession in a noisy conductance-based neuron. (A) Response of the neuron to noisy DG input $\left(\psi=110^{\circ}, \sigma_{\psi}=36^{\circ}\right)$, a noisy oscillating current conductance $\left(\sigma_{\eta}=0.01\right.$, sampling rate $\left.1 \mathrm{kHz}\right)$, and a random threshold $\left(\sigma_{\vartheta}=0.02\right)$, that is changed after each input spike. The range of assumed thresholds is depicted by the horizontal gray bar covering $\pm 3 \sigma_{\vartheta}$. In all cases, noise is gaussian. (B-D) Phase distributions for 40 repetitions of a noisy stimulus as in A. (B) The mean input phase $\psi=90^{\circ}$ leads to a bimodal distribution of phases. (C) For $\psi=110^{\circ}$, we obtain curved phase precession. (D) At $\psi=140^{\circ}$, the phase change is almost linear; see also Figure 1B. Parameters are as in Figure 9. Noise in the intracellular theta oscillation is implemented as a noisy conductance, and thus noise is low-pass-filtered by the leaky integrator. Thus, the power of noise in the membrane potential is lower than that in the current or conductance. 
5.2 Phase Precession and Simulated Place Fields. Up to now, the spatial variable in phase-place graphs as in Figure 1B has been associated with the amplitude $A$ of the mf EPSP or, equivalently, the time since the onset of periodic DG input. To juxtapose our model results with in vivo data, it is necessary to relate the EPSP amplitude $A$ to the place $x$ of a simulated rat. We therefore define the shape of a place field $G(x)$ of a DG granule cell to be gaussian with width $\sigma_{G}$ and amplitude one and periodically modulate the cell's activity to emulate the theta rhythm. Supposing that the running speed $v_{\text {run }}$ of the simulated rat is constant, we generate granule cell spikes via an inhomogeneous Poisson process with density

$$
\lambda(t)=\lambda_{0} G\left(v_{\text {run }} t\right) \sum_{n=-\infty}^{+\infty} \exp \left[-\left(t-\psi / \omega-n T_{\theta}\right)^{2} /\left(2 \sigma_{J}^{2}\right)\right]
$$

The temporal jitter $\sigma_{J}$ accounts for noise in the input phase $\psi$, as introduced earlier. We consider only the first input spike within one cycle; additional spikes generated by the process are neglected. The rate constant $\lambda_{0}$ is then chosen such that the mean number of spikes in a place field traversal is about 10 . The $\mathrm{mf}$ facilitation $\gamma_{\mathrm{mf}}$ again linearly increases with the number $f$ of granule cell spikes in a burst, that is, $\gamma_{\mathrm{mf}}^{(f)}=\gamma+f \Delta$. Using input spike trains generated in this way $\left(\psi=110^{\circ} \pm 36^{\circ}\right)$, the distribution of firing phases $\Phi$ in Figure 11A has a similar shape as the ones in the previous sections (cf. Figures 8 and 10).

In contrast to some phase distributions obtained in vivo, the total phase range of Figure 11A does not cover a whole theta cycle. This discrepancy can be resolved by including burst firing of CA3 pyramidal cells. In Figure 11B, CA3 bursts of up to three spikes in response to a mf input are generated as follows: if the leaky integrator's subthreshold membrane potential hits the firing threshold and, hence, the neuron fires a spike, we randomly assign an intraburst interval $\delta t \geq 0$ via a Poisson density

$$
\lambda_{\gamma_{\mathrm{mf}}}(\delta t)=b\left(\gamma_{\mathrm{mf}}\right) \exp \left[-b\left(\gamma_{\mathrm{mf}}\right) \delta t\right]
$$

that depends on the amplitude $\gamma_{\mathrm{mf}}$ of synaptic facilitation. For the simulation depicted in Figure 11B, we chose $b\left(\gamma_{\mathrm{mf}}\right)=2 \mathrm{~Hz}\left[\left(\gamma_{\mathrm{mf}}-\Delta\right) / \gamma\right]^{5}$, so that $b\left(\gamma_{\mathrm{mf}}\right)=2 \mathrm{~Hz}$ on the first DG input $(f=1)$. For a given randomly drawn intraburst interval $\delta t$, we use as an additional constraint that a burst is inserted into the CA3 firing pattern only if $\delta t$ is smaller than a quarter of the theta period $T_{\theta}$. The second spike then occurs $\delta t$ after the primary spike. Moreover, if $\delta t$ has been assigned a value smaller than a tenth of $T_{\theta}$, the primary spike is followed by another two spikes separated by $0.1 T_{\theta}$. The assumed dependence of bust firing on the state of facilitation yields a more frequent occurrence of CA3 bursts toward the end of the place field and, hence, for early phases. 


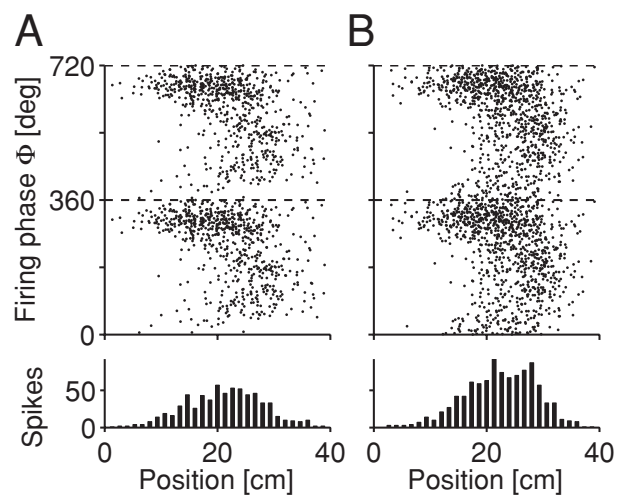

Figure 11: Phase precession in simulated place fields. The input to the conductance-based model neuron is an inhomogeneous Poisson spike train that resembles granule cell activity during the traversal of a symmetric place field. The upper panels present phase distributions for 100 simulated runs of the rat. The bottom panels depict the number of spikes as a function of position, that is, the place field of the simulated CA3 pyramidal cell. The input phase $\psi=110^{\circ}$ is fixed. (A) The phase-place distribution is bimodal, as in Figures 10B and 10C. The symmetric shape of the place field of the input is preserved in the histogram of the output spikes. (B) Burst firing in CA3 pyramidal neurons yields curved phase-place distributions covering a phase range of 360 degrees. Furthermore, the place field of the output spikes is asymmetric. In $A$ and $B$, the initial phase of the theta oscillation in each traversal was chosen randomly, simulating a variable theta phase at place field entry. The facilitation parameters $\Delta=0.0058$ and $\gamma=0.0525$ were increased compared to Figure 9 because fewer input spikes are available. The temporal jitter $\sigma_{J}$ of the firing probability density is set to $0.1 T_{\theta}$. The noise in the theta oscillation and the threshold is gaussian with $\sigma_{\eta}=0.01$ (sampling rate $1 \mathrm{kHz}$ ) and $\sigma_{\vartheta}=0.02$, respectively. Further parameters are $v_{\text {run }}=13.3 \mathrm{~cm} / \mathrm{s}, \sigma_{G}=6.67 \mathrm{~cm}, T_{\theta}=100 \mathrm{~ms}$, and $\lambda_{0}=40 \mathrm{~Hz}$. Remaining parameters are as in Figure 9: $\tau_{c}=0.075 T_{\theta}, \tau_{m}=T_{\theta}, E_{\mathrm{mf}}=2 \vartheta, \gamma_{0}=\gamma_{1}=0.014$, $E_{\theta}=-1 / 3 \vartheta, I_{\mathrm{DC}}=1.85 \vartheta / \tau_{m}$, and $V_{\text {reset }}=0.6 \vartheta$.

To conclude, by introducing a space-dependent $\mathrm{mf}$ input and burst firing in CA3 cells, we can generate asymmetric place fields with phase-place distributions that are curved (Mehta et al., 2002) and cover a total phase range of 360 degrees.

\section{Discussion}

We have provided a computational model that generates phase precession as the result of the combination of oscillations of the membrane potential of neurons and short-term facilitation of synapses (see Figure 2). Regarding phase precession of pyramidal cells in the CA3 region of the hippocampus, 
the peculiar features of the mossy fiber (mf) synapse, such as its large efficacy, huge short-term facilitation, and long time constant of decay of facilitation, are essential in this framework.

6.1 Summary of Results and Specific Predictions. The proposed model can account for features intrinsic to hippocampal phase precession that are known from the experimental literature (e.g., O'Keefe \& Recce, 1993; Skaggs et al., 1996; Harris et al., 2002; Mehta et al., 2002; Huxter et al., 2003). Here we summarize the basic properties of our model, compare them to data, and derive experimentally testable predictions.

6.1.1 Progressive Decrease of the Firing Phase. In vivo, the firing phase $\Phi$ of hippocampal pyramidal cells on average decreases during place field traversals. In the model, this phase decrease is explained by the shortterm facilitation of the mf synapse, which denotes a progressive increase of the EPSP amplitude at the CA3 pyramidal neuron. EPSP amplitudes do not markedly decrease within the time span of about 1 second that is usually needed to traverse a place field because the facilitation of the mf synapse decays with a time constant of about 10 seconds (Salin et al., 1996; Gundlfinger et al., 2007). The activity of DG granule cells is indeed characterized through spike bursts and long (tens of seconds) intervals of no activity (Jung \& McNaughton, 1993; Wiebe \& Stäubli, 1999) in which the facilitation of $\mathrm{mf}$ synapses can decay back to its baseline value and phase precession can start anew.

Let us consider the case that a rat crosses the same place field two times within a few seconds. The first traversal facilitates an associated mf synapse, which leads to phase precession. Immediately before the second traversal, this synapse is still facilitated. Our model predicts that at the beginning of the second traversal, spikes at late firing phases near 360 degrees are largely absent, and the range of phase precession is reduced. In general, interfering with the properties of facilitation of the $\mathrm{mf}$ synapse should fundamentally change phase precession in CA3.

\subsubsection{Range of Phase Precession and Preferred Firing Phase of DG Granule} Cells. For hippocampal pyramidal cells in vivo, the mean firing phase precesses from the latest phase close to 360 degrees to a phase below 180 degrees (Harris et al., 2002; Mehta et al., 2002; Huxter et al., 2003). ${ }^{1}$ The mean firing

\footnotetext{
${ }^{1}$ The theta phase is defined differently in different publications. O'Keefe and Recce (1993) could not define a particular reference phase as they did not control for the exact location of the recording electrodes. Skaggs et al. (1996) defined the theta phase $0^{\circ}$ as the maximum of the population spike activity in CA1, which they found to approximately correlate with the peak of the field EEG at the hippocampal fissure and the trough of the EEG in stratum pyramidale (sp). The EEG trough in CA1 $\mathrm{sp}$ is also taken as phase $0^{\circ}$ in Csicsvari et al. (1999), Buzsáki (2002), and Ylinen et al. (1995). Conversely, Harris et al.
} 
phase thus changes over a range of about 180 degrees, that is, less than 360 degrees. The phases of spikes, however, are distributed over the whole theta cycle. In the model, firing phases $\Phi$ close to 360 degrees are evoked by the smallest suprathreshold EPSPs, whereas the largest EPSPs trigger action potentials close to the input phase $\psi$ of DG granule cell firing, which was 90 degrees in the example shown in Figure 2. Thus, smaller input phases $\psi$ allow a larger range of firing phases $\Phi$, which extends over a range of up to $360^{\circ}-\psi$. This range refers, again, to the mean firing phase (gray lines in Figure 8), not to the distribution of spike phases (dots in Figure 8). Due to noise, the distribution of spike phases can cover 360 degrees and thus is larger than the range of phase precession (see Figures 8 and 11).

The model also demonstrates that large ranges of phase precession imply a discontinuity of the firing phase $\Phi$ as a function of the EPSP amplitude $A$, and the larger the discontinuity, the smaller is the amount of continuous phase precession (see Figures 5 and 6). Hence, there exists an input phase $\psi$ that is optimal in the sense that the range of phase precession is sufficiently large and the phase discontinuity is sufficiently small or absent.

Our model therefore predicts that the preferred phase $\psi$ of firing of DG granule cells, which is assumed to be identical to the phase of mf input to CA3, is between about 45 and 270 degrees (see Figures 5 and 6). The mean firing phase of DG granule cells in vivo in behaving animals is still a matter of debate owing to problems with spike sorting in this brain area (Jung \& McNaughton, 1993; Skaggs et al., 1996; Yamaguchi et al., 2002). On the one hand, Ylinen et al. (1995) stated that granule cells in urethane-anesthetized rats fire locked to theta oscillations at phases between 30 and 120 degrees. On the other hand, Skaggs et al. (1996) reported firing phases of dentate units to cluster around 270 degrees, with a range of spike phases of about half a theta cycle; the precession of the mean firing phase of dentate units, however, is much smaller. Phase precession in DG would boost facilitationmediated phase precession in CA3, since both contributions add up.

6.1.3 Slow Postsynaptic Integration of Pyramidal Cells. Theta oscillation periods $T_{\theta}$ in behaving animals are between about $80 \mathrm{~ms}$ and $250 \mathrm{~ms}$ (Buzsáki, 2002), which is long compared to generic membrane integration times of cortical neurons. To bridge the long temporal gap between an early input phase of DG granule cells (for example $\psi=90^{\circ}$ ) and late firing phases $\Phi \approx 360^{\circ}$ in CA3 pyramidal cells, we assumed a slow decay of EPSPs (see Figure 2B), due to a membrane integration time $\tau_{m}$ that is comparable to the

(2002) and Dragoi \& Buzsáki (2006) determined phases via a Hilbert transform of the CA1 sp EEG, which assigns phase $0^{\circ}$ to the field potential peaks. A third convention is used by Huxter et al. (2003), who take phase $0^{\circ}$ as "the + to - zero crossings" of CA1 sp theta. Again differently, Mehta et al. (2002) had no unique phase reference as they adjusted phase $0^{\circ}$ of each cell individually to achieve the best linear correlation between phase and position. 
theta period $T_{\theta}$. In vitro data indeed confirm that CA pyramidal neurons can exhibit EPSPs decaying with an extraordinary long time constant of about 100 ms (Jonas et al., 1993; Fricker \& Miles, 2000; Axmacher \& Miles, 2004).

Our model therefore predicts how phase precession depends on the theta period $T_{\theta}$. Given some fixed value of the membrane time constant $\tau_{m}$, phase precession is impossible for $T_{\theta} \gg \tau_{m}$ (see Figure 4). This may be a reason that in urethane-anesthetized animals in which the frequency of theta oscillations is typically low (about $4 \mathrm{~Hz}$; Yoder \& Pang, 2005), phase precession has not been documented.

6.1.4 Bimodality of Phase Distributions and Variability of Firing Phases. In vivo, the distribution of firing phases as a function of the animal's location can be linear, nonlinear, and also bimodal (Skaggs et al., 1996; Yamaguchi et al., 2002; see also Figure 1B). In the model, the bimodality is explained through a discontinuity of the firing phase as a function of the EPSP amplitude (see Figures 2C and 6). Nonlinear relations without a discontinuity can be obtained for input phases $\psi$ of DG granule cell spikes that are larger than some phase $\psi^{\mathrm{d}-\mathrm{c}} \geq 180^{\circ}$ (see Figures $3 \mathrm{C}$ and $5 \mathrm{~B}$ ) or by blurring the discontinuity by noise (see Figures 8,10 , and 11). We found that with increasing $\psi$, phase distributions look more and more linear.

Bimodal and nonlinear phase distributions (see Figures 1B and 11) are related to the in vivo finding that the variability of the firing phase $\Phi$ is always smallest at the entry of the place field, when the mean firing phase is close to 360 degrees, and that the phase variability is largest immediately before the animal leaves the place field, that is, when the mean firing phase is below 180 degrees (Skaggs et al., 1996; Mehta et al., 2002; Yamaguchi et al., 2002). In the framework of our model, the increase of the variability of CA3 firing phases can be attributed to a constant jitter in the input phases $\psi$ and a variable transfer of this jitter to the output spikes; for the smallest suprathreshold EPSPs with mean output phases close to 360 degrees (at the entry of a place field), the jitter in the output phase is much smaller than the jitter in the input phase (gray areas in Figures $2 \mathrm{C}$ and 7A). For large EPSPs that occur shortly before the simulated rat leaves a place field, a jitter in the input phase produces an at least equally large jitter of the output phase.

6.1.5 Discrepancies Between Model and Data. A major difficulty in comparing model results with in vivo data is that experimental procedures, setups, and data analysis are diverse. Therefore, experimentally obtained phaseplace distributions and their statistical properties are different across labs. Consequently, the phase-place distributions in Figure 11 match some experiments better than others. Our model can explain bimodal (or discontinuous) phase distributions, whereas it is difficult to produce continuous phaseplace distributions with smooth phase precession over a large fraction of the theta cycle (O'Keefe \& Recce, 1993; Mehta et al., 2002). Furthermore, our 
proposed mechanism does not directly address the observation that phase correlates better to place than to time (Huxter et al., 2003). In the next sections, we outline these and other problems in detail, and we show how the model could be extended to be consistent with the available data. After all, synaptic facilitation is not necessarily the only mechanism for phase precession; other mechanisms might also play a role, and the shape of the phaseplace distributions could thus reflect a mixture of several contributions.

6.2 Further Links to the Experimental Literature. The following features of hippocampal phase precession are in line with our model, even though we have not covered them explicitly in analytical calculations or numerical simulations in this letter. Below we briefly outline these features.

6.2.1 One- and Two-Dimensional Environments. In vivo, phase precession is seen not only in one-dimensional linear tracks but also in twodimensional open environments. In the latter, phase precession is less robust and has a smaller range on average (Skaggs et al., 1996). In both environments, however, when an animal enters a place field, the first spikes occur at the end of the theta cycle near 360 degrees (Skaggs et al., 1996; Harris et al., 2002). Our model is also consistent with these observations. In twodimensional environments, a rat often only skirts the edge of a place field. When only part of the place field is traversed, facilitation of $\mathrm{mf}$ EPSPs does not progress as far as it would during a complete traversal of the place field right through its center. Hence, the mf EPSP amplitude becomes less facilitated on average, and a smaller amount of phase precession should be detected. Nevertheless, the first spikes during a place field crossing always occur at the end of the theta cycle.

6.2.2 Multiple Place Fields. Recently, Maurer et al. (2006a) showed that a CA pyramidal neuron can exhibit more than one place field in a single environment, with each field showing a corresponding pattern of phase precession, even if the place fields of the neuron spatially overlap. This indicates that one cell could participate in different cell assemblies, coding for different locations in one environment. Assuming that several DG granule cells with different place fields provide facilitating input to one CA3 pyramidal neuron, we can explain the emergence of multiple place fields with phase precession in one CA pyramidal neuron.

6.2.3 Perturbations of the Hippocampus. Phase precession is robust to transient (up to $250 \mathrm{~ms}$ ) inactivation of the hippocampal CA region and resetting the phase of theta oscillations (Zugaro et al., 2005); after recovery from inactivation, phase precession continued, and the phase of spikes in the first theta cycle after the perturbation was more advanced than the phase of spikes just before the perturbation. These findings are consistent with our model in which the firing phase depends on the state of facilitation of the 
presynaptic terminal of the $\mathrm{mf}$ synapse. The presynapse is presumably not affected by perturbations of the activity of neurons in the CA region.

Phase precession is not prevented by the NMDA receptor antagonist CPP, which blocks experience-dependent expression of long-term potentiation and, as a result, the asymmetric expansion of hippocampal place fields during repeated route following (Mehta, Quirk, \& Wilson, 2000; Ekstrom et al., 2001). This finding is consistent with our model because plasticity at mf synapses is independent of NMDA receptors (Nicoll \& Malenka, 1995; Nicoll \& Schmitz, 2005).

6.2.4 Phase-Rate Correlations. Whether firing phase of hippocampal pyramidal cells is correlated with their instantaneous firing rate (Harris et al., 2002; Mehta et al., 2002) or not (Huxter et al., 2003) is a controversial issue. In our model, the firing phase in CA3 is determined by the amplitude of $\mathrm{mf}$ EPSCs. Since large mf EPSCs may trigger even more than one spike in a CA3 pyramidal cell, we expect a correlation between the instantaneous firing rate and the firing phase in single place field crossings. Averaging over several crossings should weaken this correlation. Furthermore, this correlation should be more pronounced in CA3 than in CA1 if a CA1 cell inherits phase precession from many CA3 cells and if the firing rate in CA1 is modulated by further excitatory as well as inhibitory inputs.

6.2.5 Nonspatial Behavior and Phase-Time Correlations. Hippocampal phase precession in rats has also been observed during wheel running and REM sleep (Harris et al., 2002). These findings are in line with our model of mf-mediated phase precession, which has no explicit spatial quality. The model thus can account for phase precession in a nonspatial context.

The shape of phase-place distributions in vivo is largely independent of running speed (Huxter et al., 2003). Averaging over many place field traversals at different running speeds therefore does not affect the phaseplace correlation but reduces the correlation between the phase of spikes and the time since the animal entered the place field (Schmidt et al., 2006). Trial-averaging thus accounts for the stronger correlation between phase and position compared to the weaker correlation between phase and time spent in the place field, as reported, for instance, by $\mathrm{O}^{\prime}$ Keefe and Recce (1993).

Because the firing rate of hippocampal pyramidal cells is roughly proportional to the running speed of rats (Ekstrom et al., 2001), the number of action potentials fired during a place field traversal is almost constant and independent of running speed (Geisler, Robbe, Zugaro, Sirota, \& Buzsáki, 2007). Similar data on the dependence of the firing rate of DG granule cells on running speed are not available. However, if the number of action potentials a DG granule cell fires during a place field traversal would be almost constant, phase precession in the framework of our model would become independent of running speed: the faster a rat runs through a place field, 
the faster the $\mathrm{mf}$ synapse facilitates and the faster the CA3 phase precesses; for a variable running speed during place field traversals, phase is then more strongly correlated with position than with time spent in the field in trial-averaged data but not in single trials.

\subsubsection{Phase Precession in the Hippocampal Formation and the Entorhinal} Cortex. Phase precession has mainly been described in CA3 and CA1 pyramidal cells, but it is less pronounced in CA1 interneurons (Maurer et al., 2006b; Ego-Stengel \& Wilson, 2007) and the dentate gyrus (Skaggs et al., 1996; Yamaguchi et al., 2002). In both latter cases, phase precession might be inherited from CA pyramidal neurons: for CA1 interneurons directly from CA1 pyramidal cells and for cells in the DG by feedback from CA3 via mossy cells.

Recently, phase precession has also been reported in grid cells of layer II in the medial entorhinal cortex (Fyhn et al., 2006). Grid cells show several firing fields covering the environment like a grid. Fyhn et al. found a full cycle of phase precession in each firing field, indicating that phase precession might be generated in the entorhinal cortex (EC) or at an earlier stage. There, phase precession could be generated through interaction of shortterm facilitation and membrane potential oscillation in a similar way as we have proposed for the $\mathrm{mf}$ synapse in CA3.

Could phase precession in the DG and the CA region be inherited from the EC (McNaughton, Battaglia, Jensen, Moser, \& Moser, 2006)? One objection against inheritance is the high convergence from EC to DG and CA, that is, cells in DG and CA receive input from a large number of EC cells. To inherit phase precession, the EC cells projecting to one postsynaptic neuron need to show similar phase precession, which requires considerable finetuning of the connectivity. Otherwise, phase precession would be severely reduced by averaging over many cells firing at different phases. A further argument against inheritance of phase precession from EC to CA via the perforant path is that the corresponding synapses are located at the distal parts of the dendritic tree of pyramidal cells. These synapses are relatively weak and thus probably not suited to reliably transfer precise spike timing (Jarsky, Roxin, Kath, \& Spruston, 2005).

6.3 Comparison with Other Models. In this letter we explain how phase precession can be generated in single cells, which is similar to the approaches of O'Keefe and Recce (1993), Kamondi et al. (1998), Magee (2001), Harris et al. (2002), Mehta et al. (2002), Lengyel et al. (2003), and Huhn et al. (2005). Some of the single cell models assume that precession arises in CA1 (Kamondi et al., 1998; Magee, 2001; Harris et al., 2002; Mehta et al., 2002). Another class of phase precession models explicitly requires a network of neurons. Some of these network models suggest the origin of phase precession to be in CA3 (Tsodyks et al., 1996; Wallenstein \& Hasselmo, 1997; Bose \& Recce, 2001; Booth \& Bose, 2001; Scarpetta \& Marinaro, 2005) and 
others in the entorhinal cortex (Yamaguchi, 2003; Hasselmo \& Eichenbaum, 2005). Finally, Jensen and Lisman (1996), Hasselmo et al. (2002), Koene et al. (2003), and Lisman et al. (2005) predict phase precession to be generated through an interplay of the entorhinal cortex and CA3.

Mechanisms used to explain the generation of phase precession include (1) the interaction of two oscillators with slightly different frequencies (O'Keefe \& Recce, 1993; Kamondi et al., 1998; Bose \& Recce, 2001; Booth \& Bose, 2001; Lengyel et al., 2003; Yamaguchi, 2003; Huhn et al., 2005), (2) the asymmetry of place fields (Mehta et al., 2000, 2002), (3) the adaptation of the spiking activity of pyramidal cells in response to sustained excitatory input (Kamondi et al., 1998; Magee, 2001; Harris et al., 2002; Mehta et al., 2002; Mehta, Quirk, \& Wilson, 2000) and (4) the use of a short-term memory buffer (Hasselmo et al., 2002; Koene et al., 2003); see, for example, Zugaro et al. (2005), Maurer and McNaughton (2007), and Yamaguchi et al. (2007) for a discussion of phase precession models. It is also conceivable that phase precession is generated through an interaction of two or more mechanisms.

Mechanistic models of phase precession can also be divided into models that explain this phenomenon as the result of (sequence) learning (Tsodyks et al., 1996; Jensen \& Lisman, 1996; Wallenstein \& Hasselmo, 1997; Hasselmo et al., 2002; Koene et al., 2003; Mehta et al., 2002; Scarpetta \& Marinaro, 2005; Hasselmo \& Eichenbaum, 2005; Jensen \& Lisman, 2005), and models, including ours, that do not require previous (sequence) learning (O'Keefe \& Recce, 1993; Magee, 2001; Harris et al., 2002; Lengyel et al., 2003; Yamaguchi, 2003; Huhn et al., 2005). The latter retrospective models have the advantage that phase precession in CA1 can occur in the first exposure to a new environment, which is in accordance with the observations of Rosenzweig, Ekstrom, Redish, McNaughton, and Barnes (2000), Mehta et al. (2002), and Chen and Frank (2007). An argument in favor of retrospective models is the avoidance of a combinatorial explosion. In other words, if phase precession is a result of a learned trajectory in a two-dimensional environment, this phenomenon should not be observed if the rat enters a direction-insensitive place field from a different new direction. Learning phase precession separately for many different trajectories, however, demands a high amount of plasticity resources.

6.4 Limitations and Outlook. Our model is mostly restricted to a single CA3 spike per cycle. We think of it as the first spike of an intracycle burst. Multiple firing has been included using a phenomenological model. Mechanistic explanations could arise from, for example, intrinsic cell properties, granule cell bursts, or recurrent activity.

A further extension to our model could be the inclusion of a more realistic theta fine structure since the phase of firing is sensitively modulated by the time course of the intracellular oscillations (Hopfield, 1995). The model predicts that the distribution of spike phases is specifically altered by changes in the oscillation amplitude, the mean level of 
depolarization, the theta frequency, and the shape of the theta oscillation. In particular, skewed or sawtooth-like oscillations could alter the range of continuous phase precession. We have refrained from adding further details to the model and instead focused on the principal mechanism of synaptic facilitation.

How phase precession is adapted to the particular size of a place field of a CA3 place cell is another open issue. Place cells with small place fields exhibit rapid phase precession, whereas cells with large fields display slow phase precession such that the range of firing phases is the same in both cases (Ekstrom et al., 2001; Dragoi \& Buzsáki, 2006; Geisler et al., 2007). Mossy fiber facilitation should therefore be strong for small place fields where a CA3 place cell receives only few DG input spikes on average. Conversely, for large place fields in which a CA3 place cell receives a large number of DG spikes, facilitation should be weak. Moreover, the smallest mf EPSPs should ideally be subthreshold so that occasional spontaneous DG spikes do not drive CA3 cells. Such an adaptation of $\mathrm{mf}$ facilitation might be achieved through long-term potentiation (LTP) and long-term depression (LTD) of the synapse (Nicoll \& Malenka, 1995; Nicoll \& Schmitz, 2005; Gundlfinger et al., 2007). As expected for a mechanism of input adaptation, induction of $\mathrm{mf}$ LTP and LTD occurs predominantly presynaptically (Nicoll \& Schmitz, 2005); plasticity is mainly triggered by the activity of the DG granule cell and to a much lesser extent depends on the activity of the CA3 pyramidal cell. To summarize, experiencedependent variations of the size of a place field and changes in the speed of phase precession are tightly coupled (Chen \& Frank, 2007). Both phenomena might therefore be based on a common mechanism that is related to experience-dependent changes of properties of short-term facilitation (Gundlfinger et al., 2007).

Taken together, we suggest that synaptic facilitation (e.g., at hippocampal mossy fiber synapses) generates the phenomenon of phase precession, which is a temporal spike code. Hippocampal phase precession might be important in sequence learning (Skaggs et al., 1996; Mehta et al., 2002; Sato \& Yamaguchi, 2003; Melamed et al., 2004; Jensen \& Lisman, 2005; Lengyel et al., 2005). Our model thus provides a potential link between the physiology of the mossy fiber synapse and behavior (Lipp, Schwegler, Heimrich, \& Driscoll, 1988). This is the more so as DG granule cells are necessary for normal spatial learning but not for place field firing in the CA (McNaughton, Barnes, Meltzer, \& Sutherland, 1989).

\section{Appendix A: Analytical Solutions to the Threshold Model} for $\tau_{c}=0$

Assuming exponentially decaying EPSPs with instantaneous rise $\left(\tau_{c}=0\right)$, we can analytically solve the threshold model as defined in section 3 . Here 
we derive the formulas dealt with in section 3.2, which quantitatively describe phase precession.

We briefly repeat the definition of the threshold model, where EPSPs always start from zero resting potential; that is, only isolated EPSPs are considered. An EPSP $\epsilon$ that is elicited at phase $\psi$ triggers a spike at the phase $\Phi$ at which the firing threshold $\vartheta>0$ is reached for the first time. In other words, the condition

$$
\vartheta(\Phi)=\epsilon(\Phi-\psi)
$$

has to be fulfilled. The theta modulated firing threshold $\vartheta$ is defined as

$$
\vartheta(\varphi)=\vartheta_{0}-\vartheta_{1} \cos \varphi
$$

with the mean threshold $\vartheta_{0}$ and the amplitude $\vartheta_{1}$ of the threshold oscillation. We require $\vartheta_{0}>\vartheta_{1}>0$. The theta phase $\varphi=\omega t$ is determined by the time $t$ and the circular frequency $\omega=2 \pi / T_{\theta}$, where $T_{\theta}$ is the theta period. The shape of an exponentially decaying EPSP $\epsilon$ with instantaneous rise is given by

$$
\epsilon(\varphi)=A \exp \left[-\varphi /\left(\omega \tau_{m}\right)\right] \Theta(\varphi)
$$

where $A$ is the amplitude and $\tau_{m}>0$ is the membrane time constant, that is, the time constant of the decay. The function $\Theta(x)$ denotes the Heaviside step function, $\Theta(x)=1$ for $x \geq 0$, and $\Theta(x)=0$ otherwise.

A.1 Maximum Firing Phase $\boldsymbol{\Phi}^{\max }$. The maximum firing phase $\Phi^{\max }$ is assumed for decaying EPSPs $\epsilon$ that are just large enough to reach a decreasing threshold $\vartheta$. To calculate $\Phi^{\max }$, we require that $\epsilon$ and $\vartheta$ touch at a point that is characterized by two conditions. First, the values of $\epsilon$ and $\vartheta$ are equal, $\epsilon\left(\Phi^{\max }-\psi\right)=\vartheta\left(\Phi^{\max }\right)$. Second, the slopes of $\epsilon$ and $\vartheta$ are identical, $\epsilon^{\prime}\left(\Phi^{\max }-\psi\right)=\vartheta^{\prime}\left(\Phi^{\max }\right)$, and negative (see also Figure 3A). Mathematically, $\Phi^{\max }$ can be obtained by using equations A.2, A.3, and $\epsilon^{\prime}\left(\Phi^{\max }-\psi\right)=\vartheta^{\prime}\left(\Phi^{\max }\right)$ in equation A.1, which leads to

$$
\frac{1}{\omega \tau_{m}}\left[\vartheta_{1} \cos \left(\Phi^{\max }\right)-\vartheta_{0}\right]=\vartheta_{1} \sin \left(\Phi^{\max }\right)
$$

Defining the theta modulation depth $\rho=\vartheta_{1} / \vartheta_{0}>0$, we can rewrite equation A.4 as

$$
\rho^{-1}=\cos \left(\Phi^{\max }\right)-\omega \tau_{m} \sin \left(\Phi^{\max }\right)
$$


Using $\tan \alpha=1 /\left(\omega \tau_{m}\right)$ we find

$$
\rho^{-1}=-\sqrt{1+\left(\omega \tau_{m}\right)^{2}} \sin \left(\Phi^{\max }-\alpha\right)
$$

Equations A.4 to A.6, are subject to two further constraints. First, the curvature of $\vartheta$ is larger than the one of $\epsilon$ since the EPSP has to reach the threshold from below. Hence, the second derivatives obey $\epsilon\left(\Phi^{\max }-\psi\right)^{\prime \prime}<$ $\vartheta\left(\Phi^{\max }\right)^{\prime \prime}$. Since we have $0<\epsilon\left(\Phi^{\max }-\psi\right)^{\prime \prime}$ for exponentially decaying EPSPs, $\vartheta\left(\Phi^{\max }\right)^{\prime \prime}=\vartheta_{1} \cos \left(\Phi^{\max }\right)$ from equation A.2, and $\vartheta_{1}>0$ by definition, we find $\cos \Phi^{\max }>0$ and $0 \leq \Phi^{\max }<\pi / 2$ or $3 / 2 \pi<\Phi^{\max } \leq 2 \pi$. Second, a decaying threshold means $0>\vartheta^{\prime}\left(\Phi^{\max }\right)=\vartheta_{1} \sin \left(\Phi^{\max }\right)$. The two constraints therefore imply that $\left.\left.\Phi^{\max } \in\right] 3 / 2 \pi, 2 \pi\right]$. Taking the appropriate branch of the inverse sine in equation A.6, we find the maximum firing phase to be

$$
\Phi^{\max }=2 \pi-\arcsin \left\{\left[\rho \sqrt{1+\left(\omega \tau_{m}\right)^{2}}\right]^{-1}\right\}+\arctan \left[1 /\left(\omega \tau_{m}\right)\right]
$$

for $\rho \sqrt{1+\left(\omega \tau_{m}\right)^{2}} \geq 1$. Equation A.7 equals equation 3.5 in section 3.2.2.

\section{A.2 Switch from Discontinuous to Continuous Phase Precession at} Phase $\psi^{\mathrm{d}-\mathrm{c}}$. The phase $\psi^{\mathrm{d}-\mathrm{c}}$ separates the regions of discontinuous and continuous phase precession. Similar to the previous paragraph, the phase $\psi^{\mathrm{d}-\mathrm{c}}$ is determined by two conditions: first, $\epsilon\left(\psi^{\mathrm{d}-\mathrm{c}}-\psi\right)=\vartheta\left(\psi^{\mathrm{d}-\mathrm{c}}\right)$, and second, $\epsilon^{\prime}\left(\psi^{\mathrm{d}-\mathrm{c}}-\psi\right)=\vartheta^{\prime}\left(\psi^{\mathrm{d}-\mathrm{c}}\right)$. In analogy to equation A.4, we find

$$
\frac{1}{\omega \tau_{m}}\left[\vartheta_{1} \cos \left(\psi^{\mathrm{d}-\mathrm{c}}\right)-\vartheta_{0}\right]=\vartheta_{1} \sin \left(\psi^{\mathrm{d}-\mathrm{c}}\right) .
$$

In contrast to the previous paragraph, however, the curvatures of $\vartheta$ and $\epsilon$ need to have different signs (see Figure $3 C$ ). We still seek values of $\psi^{\mathrm{d}-\mathrm{c}}$ in the decaying part of the threshold. The constraints thus imply that $\psi^{\mathrm{d}-\mathrm{c}} \in$ ]$\pi, 3 / 2 \pi$ [. Taking the appropriate branch of the inverse sine in equation A.8, we arrive at

$$
\psi^{\mathrm{d}-\mathrm{c}}=\pi+\arcsin \left\{\left[\rho \sqrt{1+\left(\omega \tau_{m}\right)^{2}}\right]^{-1}\right\}+\arctan \left[1 /\left(\omega \tau_{m}\right)\right]
$$

for $\rho \sqrt{1+\left(\omega \tau_{m}\right)^{2}} \geq 1$. Equation A.9 equals equation 3.6 in section 3.2.3.

\section{Appendix B: Singularities of the Threshold Model for $\tau_{c}>0$}

The results derived in appendix A can be generalized to EPSPs $\epsilon(\varphi-\psi)=$ $A k(\varphi)$ with arbitrary shape $k \geq 0$ and constant input phase $\psi$. One therefore defines the firing phase $\Phi$ through the implicit equation

$$
G(\Phi, A)=A k(\Phi)-\vartheta(\Phi) \equiv 0
$$


in which $\vartheta$ is the theta-modulated voltage threshold from equation A.2. If the phase $\Phi$ smoothly precesses, $\Phi(A)$ is differentiable such that $G(\Phi(A), A) \equiv 0$. Differentiation then yields

$$
\frac{\partial G}{\partial \Phi} \frac{\mathrm{d}}{\mathrm{d} A} \Phi=-\frac{\partial G}{\partial A}=-k(\Phi)<0,
$$

where the last inequality is due to $k(\Phi)>0$ for a positive firing threshold $\vartheta(\Phi)>0$. Consequently, $\Phi(A)$ is singular at $\Phi^{\mathrm{s}}$ if and only if

$$
0=\left.\frac{\partial G}{\partial \Phi}\right|_{\Phi=\Phi^{\mathrm{s}}}=A k^{\prime}\left(\Phi^{\mathrm{s}}\right)-\vartheta_{1} \sin \left(\Phi^{\mathrm{s}}\right) .
$$

Combining equations B.1 and B.2 with $\rho=\vartheta_{1} / \vartheta_{0}$ then leads to

$$
\rho^{-1}=\cos \left(\Phi^{\mathrm{s}}\right)+\frac{k\left(\Phi^{\mathrm{s}}\right)}{k^{\prime}\left(\Phi^{\mathrm{s}}\right)} \sin \left(\Phi^{\mathrm{s}}\right),
$$

which is reminiscent of equations A.5 and A.8 that determine the characteristic phases $\Phi^{\max }$ and $\psi^{\mathrm{d}-\mathrm{c}}$, respectively, for exponentially decaying EPSPs. In contrast, equation B.3 cannot be solved explicitly for arbitrary $k$, and the singular phases $\Phi^{\mathrm{s}}$ have to be determined numerically. We consider an EPSP shape $k$ as generic if equation B.3 provides not more than two singular phases, that is, a maximum firing phase and at most one further discontinuity.

\section{Acknowledgments}

We thank Robert Schmidt for comments on the manuscript and valuable discussions. This study was supported by grants from the Deutsche Forschungsgemeinschaft (Emmy Noether fellowship to R.K., grant number Ke 788/1-3,4; Sonderforschungsbereich 618 "Theoretical Biology") and by a grant from the Bundesministerium für Bildung und Forschung to R.K. and D.S. (grant number 01GQ0410).

\section{References}

Abbott, L. F., \& Regehr, W. R. (2004). Synaptic computation. Nature, 431, 796-803.

Abbott, L. F., Varela, J. A., Sen, K., \& Nelson, S. B. (1997). Synaptic depression and cortical gain control. Science, 275, 220-224.

Artun, Ö. B., Shouval, H. Z., \& Cooper, L. N. (1998). The effect of dynamic synapses on spatiotemporal receptive fields in visual cortex. Proc. Natl. Acad. Sci. USA, 95, 11999-12003.

Axmacher, N., \& Miles, R. (2004). Intrinsic cellular currents and the temporal precision of EPSP-action potential coupling in CA1 pyramidal cells. J. Physiol., 555, 713-725. 
Bland, B. H., Konopacki, J., \& Dyck, R. (2005). Heterogeneity among hippocampal pyramidal neurons revealed by their relation to theta-band oscillation and synchrony. Exp. Neurol., 195, 458-474.

Booth, V., \& Bose, A. (2001). Neural mechanisms for generating rate and temporal codes in model CA3 pyramidal cells. J. Neurophysiol., 85, 2432-2445.

Bose, A., \& Recce, M. (2001). Phase precession and phase-locking of hippocampal pyramidal cells. Hippocampus, 11, 204-215.

Brun, V. H., Otnass, M. K., Molden, S., Steffenach, H. A., Witter, M. P., Moser, M. B., et al. (2002). Place cells and place recognition maintained by direct entorhinalhippocampal circuitry. Science, 296, 2243-2246.

Buchs, N. J., \& Senn, W. (2002). Spike-based synaptic plasticity and the emergence of direction selective simple cells: Simulation results. J. Comput. Neurosci., 13, 167186.

Buonomano, D. V., \& Merzenich, M. M. (1995). Temporal information transformed into a spatial code by a neural network with realistic properties. Science, 267, 1028-1030.

Buzsáki, G. (2002). Theta oscillations in the hippocampus. Neuron, 33, 325-340.

Chen, S., \& Frank, L. M. (2007). Experience-dependent dynamics of spatiotemporal precision and synchrony in place cells. In Proceedings of the 4th Computational and Systems Neuroscience Meeting, III-49 (p. 251). Available online at http:/ / cosyne.org.

Csicsvari, J., Hirase, H., Czurkó, A., Mamiya, A., \& Buzsáki, G. (1999). Oscillatory coupling of hippocampal pyramidal cells and interneurons in the behaving rat. J. Neurosci., 19, 274-287.

Dragoi, G., \& Buzsáki, G. (2006). Temporal encoding of place sequences by hippocampal cell assemblies. Neuron, 50, 145-157.

Ego-Stengel, V., \& Wilson, M. A. (2007). Spatial selectivity and theta phase precession in CA1 interneurons. Hippocampus, 17, 161-174.

Ekstrom, A. D., Meltzer, J., McNaughton, B. L., \& Barnes, C. A. (2001). NMDA receptor antagonism blocks experience-dependent expansion of hippocampal "place fields". Neuron, 31, 631-638.

Fortin, N. J., Agster, K. L., \& Eichenbaum, H. B. (2002). Critical role of the hippocampus in memory for sequences of events. Nat. Neurosci., 5, 458-462.

Fricker, D., \& Miles, R. (2000). EPSP amplification and the precision of spike timing in hippocampal neurons. Neuron, 28, 559-569.

Fyhn, M., Hafting, T., Moser, M. B., \& Moser, E. I. (2006). Theta modulation and phase precession in grid cells in the medial entorhinal cortex. FENS Abstr., 3, A197.30.

Geisler, C., Robbe, D., Zugaro, M., Sirota, A., \& Buzsáki, G. (2007). Hippocampal place cell assemblies are speed-controlled oscillators. Proc. Natl. Acad. Sci. USA, 104, 8149-8154.

Gundlfinger, A., Leibold, C., Gebert, K., Moisel, M., Schmitz, D., \& Kempter, R. (2007). Differential modulation of short-term synaptic dynamics by long-term potentiation at mouse hippocampal mossy fibre synapse. J. Physiol., 585.3, 853865.

Harris, K. D., Henze, D. A., Hirase, H., Leinekugel, X., Dragoi, G., Czurko, A., et al. (2002). Spike train dynamics predicts theta-related phase precession in hippocampal pyramidal cells. Nature, 417, 738-741. 
Hasselmo, M. E., Canon, R. C., \& Koene, R. (2002). A simulation of parahippocampal and hippocampal structures guiding spatial navigation of a virtual rat in a virtual environment: A functional framework for theta theory. In M. P. Witter \& F. G. Wouterlood (Eds.), The parahippocampal region: Organization and role in cognitive functions (pp. 139-161). New York: Oxford University Press.

Hasselmo, M. E., \& Eichenbaum, H. (2005). Hippocampal mechanisms for the context-dependent retrieval of episodes. Neural Netw., 18, 1172-1190.

Henze, D. A., Urban, N. N., \& Barrionuevo, G. (2000). The multifarious hippocampal mossy fiber pathway: A review. Neuroscience, 98, 407-427.

Henze, D. A., Wittner, L., \& Buzsáki, G. (2002). Single granule cells reliably discharge targets in the hippocampal CA3 network in vivo. Nat. Neurosci., 5, 790-795.

Hopfield, J. J. (1995). Pattern recognition computation using action potential timing for stimulus representation. Nature, 376, 33-36.

Huhn, Z., Orban, G., Erdi, P., \& Lengyel, M. (2005). Theta oscillation-coupled dendritic spiking integrates input on a long time scale. Hippocampus, 15, 950962.

Huxter, J., Burgess, N., \& O'Keefe, J. (2003). Independent rate and temporal coding in hippocampal pyramidal cells. Nature, 425, 828-832.

Jarsky, T., Roxin, A., Kath, W. L., \& Spruston, N. (2005). Conditional dendritic spike propagation following distal synaptic activation of hippocampal CA1 pyramidal neurons. Nat. Neurosci., 8, 1667-1676.

Jensen, O., \& Lisman, J. E. (1996). Hippocampal CA3 region predicts memory sequences: Accounting for the phase precession of place cells. Learn. Mem., 3, 279287.

Jensen, O., \& Lisman, J. E. (2005). Hippocampal sequence-encoding driven by a cortical multi-item working memory buffer. Trends Neurosci., 28, 67-72.

Jonas, P., Major, G., \& Sakmann, B. (1993). Quantal components of unitary EPSCs at the mossy fiber synapse on CA3 pyramidal cells of rat hippocampus. J. Physiol., 472, 615-663.

Jung, M. W., \& McNaughton, B. L. (1993). Spatial selectivity of unit activity in the hippocampal granular layer. Hippocampus, 3, 165-182.

Kamondi, A., Acsady, L., Wang, X. J., \& Buzsáki, G. (1998). Theta oscillations in somata and dendrites of hippocampal pyramidal cells in vivo: Activity-dependent phase-precession of action potentials. Hippocampus, 8, 244-261.

Kesner, R. P., Gilbert, P. E., \& Barua, L. A. (2002). The role of the hippocampus in memory for the temporal order of a sequence of odors. Behav. Neurosci., 116, 286-290.

Koene, R. A., Gorchetchnikov, A., Cannon, R. C., \& Hasslemo, M. E. (2003). Modeling goal-directed spatial navigation in the rat based on physiological data from the hippocampal formation. Neural Networks, 16, 577-584.

Lee, A. K., Manns, I. D., Sakmann, B., \& Brecht, M. (2006). Whole-cell recordings in freely moving rats. Neuron, 51, 399-407.

Lengyel, M., Huhn, Z., \& Erdi, P. (2005). Computational theories on the function of theta oscillations. Biol. Cybern., 92, 393-408.

Lengyel, M., Szatmary, Z., \& Erdi, P. (2003). Dynamically detuned oscillations account for the coupled rate and temporal code of place cell firing. Hippocampus, 13, 700-714. 
Lipp, H. P., Schwegler, H., Heimrich, B., \& Driscoll, P. (1988). Infrapyramidal mossy fibers and two-way avoidance learning: Developmental modification of hippocampal circuitry and adult behavior of rats and mice. J. Neurosci., 8, 19051921.

Lisman, J. E., Talamini, L. M., \& Raffone, A. (2005). Recall of memory sequences by interaction of the dentate and CA3: A revised model of the phase precession. Neural Netw., 18, 1191-1201.

Maass, W., Natschläger, T., \& Markram, H. (2002). Real-time computing without stable states: A new framework for neural computation based on perturbations. Neural Comput., 14, 2531-2560.

Magee, J. C. (2001). Dendritic mechanisms of phase precession in hippocampal CA1 pyramidal neurons. J. Neurophysiol., 86, 528-532.

Maurer, A. P., Cowen, S. L., Burke, S. N., Barnes, C. A., \& McNaughton, B. L. (2006a). Organization of hippocampal cell assemblies based on theta phase precession. Hippocampus, 16, 785-794.

Maurer, A. P., Cowen, S. L., Burke, S. N., Barnes, C. A., \& McNaughton, B. L. (2006b). Phase precession in hippocampal interneurons showing strong functional coupling to individual pyramidal cells. J. Neurosci., 26, 13485-13492.

Maurer, A. P., \& McNaughton, B. L. (2007). Network and intrinsic cellular mechanisms underlying theta phase precession of hippocampal neurons. Trends Neurosci., 30, 325-333.

McNaughton, B. L., Barnes, C. A., Meltzer, J., \& Sutherland, R. J. (1989). Hippocampal granule cells are necessary for normal spatial learning but not for spatiallyselective pyramidal cell discharge. Exp. Brain Res., 76, 485-496.

McNaughton, B. L., Battaglia, F. P., Jensen, O., Moser, E. I., \& Moser, M. B. (2006). Path integration and the neural basis of the "cognitive map." Nat. Rev. Neurosci., 7, 663-678.

McNaughton, B. L., \& Morris, R. G. M. (1987). Hippocampal synaptic enhancement and information storage within a distributed memory system. Trends Neurosci., 10, 408-415.

Melamed, O., Gerstner, W., Maass, W., Tsodyks, M., \& Markram, H. (2004). Coding and learning of behavioural sequences. Trends Neurosci., 27, 11-14.

Mehta, M. R., Lee, A. K., \& Wilson, M. A. (2002). Role of experience and oscillations in transforming a rate code into a temporal code. Nature, 417, 741-746.

Mehta, M. R., Quirk, M. C., \& Wilson, M. A. (2000). Experience-dependent, asymmetric shape of hippocampal receptive fields. Neuron, 25, 707-715.

Nicoll, R. A., \& Malenka, R. C. (1995). Contrasting properties of two forms of longterm potentiation in the hippocampus. Nature, 377, 115-118.

Nicoll, R., \& Schmitz, D. (2005). Synaptic plasticity at hippocampal mossy fibre synapses. Nat. Rev. Neurosci., 6, 863-876.

O'Keefe, J., \& Dostrovsky, J. (1971). The hippocampus as a spatial map: Preliminary evidence from unit activity in the freely-moving rat. Brain Res., 34, 171175.

O'Keefe, J., \& Recce, M. L. (1993). Phase relationship between hippocampal place units and EEG theta rhythm. Hippocampus, 3, 317-330.

Rosenzweig, E. S., Ekstrom, A. D., Redish, A. D., McNaughton, B. L., \& Barnes, C. A. (2000). Phase precession as an experience independent process: 
Hippocampal pyramidal cell phase precession in a novel environment and under NMDA-receptor blockade. Abstr. Soc. Neurosci., 26, 982.

Salin, P. A., Scanziani, M., Malenka, R. C., \& Nicoll, R. A. (1996). Distinct short-term plasticity at two excitatory synapses in the hippocampus. Proc. Natl. Acad. Sci. USA, 93, 13304-13309.

Sato, N., \& Yamaguchi, Y. (2003). Memory encoding by theta phase precession in the hippocampal network. Neural Comput., 15, 2379-2397.

Scarpetta, S., \& Marinaro, M. (2005). A learning rule for place fields in a cortical model: Theta phase precession as a network effect. Hippocampus, 15, 979-989.

Schmidt, R., Leibold, C., Thurley, K., Gundlfinger, A., Ritz, R., Schmitz, D., \& Kempter, R. (2006). Theta-frequency dependence and phase-place correlations in models of hippocampal phase precession. Abstr. Soc. Neurosci., 17, 371.

Senn, W., Segev, I., \& Tsodyks, M. V. (1998). Reading neuronal synchrony with depressing synapses. Neural Comput., 10, 815-819.

Silva, A. J., Rosahl, T. W., Chapman, P. F., Marowitz, Z., Friedman, E., Frankland, P. W., et al. (1996). Impaired learning in mice with abnormal short-lived plasticity. Curr. Biol., 6, 1509-1518.

Skaggs, W. E., McNaughton, B. L., Wilson, M. A., \& Barnes, C. A. (1996). Theta phase precession in hippocampal neuronal populations and the compression of temporal sequences. Hippocampus, 6, 149-172.

Toth, K., Suares, G., Lawrence, J. J., Philips-Tansey, E., \& McBain, C. J. (2000). Differential mechanisms of transmission at three types of mossy fiber synapse. $J$. Neurosci., 20, 8279-8289.

Treves, A., \& Rolls, E. T. (1992). Computational constraints suggest the need for two distinct input systems to the hippocampal CA3 network. Hippocampus, 2, 189199.

Troyer, T. W., \& Miller, K. D. (1997). Physiological gain leads to high ISI variability in a simple model of a cortical regular spiking cell. Neural Comput., 9, 971983.

Tsodyks, M. V., \& Markram, H. (1997). The neural code between neocortical pyramidal neurons depends on neurotransmitter release probability. Proc. Natl. Acad. Sci. USA, 94, 719-723.

Tsodyks, M. V., Skaggs, W. E., Sejnowski, T. J., \& McNaughton, B. L. (1996). Population dynamics and theta phase precession of hippocampal place cell firing: A spiking neuron model. Hippocampus, 6, 271-280.

Urban, N. N., Henze, D. A., \& Barrionuevo, G. (2001). Revisiting the role of the hippocampal mossy fiber synapse. Hippocampus, 11, 408-417.

Wallenstein, G. V., \& Hasselmo, M. E. (1997). GABAergic modulation of hippocampal population activity: Sequence learning, place field development and the phase precession effect. J. Neurophysiol., 78, 393-408.

Wiebe, S. P., \& Stäubli, U. V. (1999). Dynamic filtering of recognition memory codes in the hippocampus. J. Neurosci., 19, 10562-10574.

Yamaguchi, Y. (2003). A theory of hippocampal memory based on theta phase precession. Biol. Cybern., 89, 1-9.

Yamaguchi, Y., Aota, Y., McNaughton, B. L., \& Lipa, P. (2002). Bimodality of theta phase precession in hippocampal place cells in freely running rats. J. Neurophysiol., 87, 2629-2642. 
Yamaguchi, Y., Sato, N., Wagatsuma, H., Wu, Z., Molter, C., \& Aota, Y. (2007). A unified view of theta-phase coding in the entorhinal-hippocampal system. Curr. Opin. Neurobiol., 17, 197-204.

Ylinen, A., Soltesz, I., Bragin, A., Penttonen, M., Sik, A., \& Buzsáki, G. (1995). Intracellular correlates of hippocampal theta rhythm in identified pyramidal cells, granule cells and basket cells. Hippocampus, 5, 78-90.

Yoder, R. M., \& Pang, K. C. (2005). Involvement of GABAergic and cholinergic medial septal neurons in hippocampal theta rhythm. Hippocampus, 15, 381-392.

Zucker, R. S., \& Regehr, W. G. (2002). Short-term synaptic plasticity. Annu. Rev. Neurosci., 64, 355-405.

Zugaro, M. B., Monconduit, L., \& Buzsáki, G. (2005). Spike phase precession persists after transient intrahippocampal perturbation. Nat. Neurosci., 8, 67-71.

Received July 27, 2006; accepted June 29, 2007. 
Copyright of Neural Computation is the property of MIT Press and its content may not be copied or emailed to multiple sites or posted to a listserv without the copyright holder's express written permission. However, users may print, download, or email articles for individual use. 